\title{
THE KECK + MAGELLAN SURVEY FOR LYMAN LIMIT ABSORPTION. III. SAMPLE DEFINITION AND COLUMN DENSITY MEASUREMENTS
}

\author{
J. Xavier Prochaska ${ }^{1}$, John M. O’Meara ${ }^{2}$, Michele Fumagalli ${ }^{3,4}$, Rebecca A. Bernstein ${ }^{4}$, and Scott M. Burles ${ }^{5}$ \\ ${ }^{1}$ Department of Astronomy and Astrophysics, UCO/Lick Observatory, University of California, 1156 High Street, Santa Cruz, CA 95064, USA \\ ${ }^{2}$ Department of Chemistry and Physics, Saint Michael's College, One Winooski Park, Colchester, VT 05439, USA \\ ${ }^{3}$ Institute for Computational Cosmology, Department of Physics, Durham University, South Road, Durham, DH1 3LE, UK \\ ${ }^{4}$ Observatories of the Carnegie Institution for Science, 813 Santa Barbara Street, Pasadena, CA 91101, USA \\ ${ }^{5}$ Cutler Group, LP., 101 Montgomery Street, San Francisco, CA 94104, USA \\ Received 2015 January 28; accepted 2015 June 4; published 2015 October 6
}

\begin{abstract}
We present an absorption-line survey of optically thick gas clouds—Lyman Limit Systems (LLSs)—observed at high dispersion with spectrometers on the Keck and Magellan telescopes. We measure column densities of neutral hydrogen $N_{\mathrm{HI}}$ and associated metal-line transitions for 157 LLSs at $z_{\mathrm{LLS}}=1.76-4.39$ restricted to $10^{17.3} \mathrm{~cm}^{-2} \leqslant N_{\mathrm{HI}}<10^{20.3} \mathrm{~cm}^{-2}$. An empirical analysis of ionic ratios indicates an increasing ionization state of the gas with decreasing $N_{\mathrm{HI}}$ and that the majority of LLSs are highly ionized, confirming previous expectations. The $\mathrm{Si}^{+} / \mathrm{H}^{0}$ ratio spans nearly four orders of magnitude, implying a large dispersion in the gas metallicity. Fewer than 5\% of these LLSs have no positive detection of a metal transition; by $z \sim 3$, nearly all gas that is dense enough to exhibit a very high Lyman limit opacity has previously been polluted by heavy elements. We add new measurements to the small subset of LLS $(\approx 5 \%-10 \%)$ that may have super-solar abundances. High $\mathrm{Si}^{+} / \mathrm{Fe}^{+}$ratios suggest an $\alpha$-enhanced medium, whereas the $\mathrm{Si}^{+} / \mathrm{C}^{+}$ratios do not exhibit the super-solar enhancement inferred previously for the Ly $\alpha$ forest.
\end{abstract}

Key words: intergalactic medium

Supporting material: figure set, machine-readable tables

\section{INTRODUCTION}

As a packet of ionizing radiation $(h \nu \geqslant 1 \mathrm{Ryd})$ traverses the universe, it has a high probability of encountering a slab of optically thick $\mathrm{H}_{\mathrm{I}}$ gas. For sources in the $z \sim 4$ universe, the mean free path is only $\approx 30 \mathrm{Mpc}$ (physical; Worseck et al. 2014), i.e., less than $2 \%$ of the event horizon. Observationally, researchers refer to this optically thick gas as Lyman limit systems (LLSs) owing to their unmistakable signature of continuum opacity at the Lyman limit $(\approx 912 \AA)$ in the system restframe. A fraction of this gas lies within the dense, neutral interstellar medium (ISM) of galaxies, yet the majority of the opacity must arise from gas outside the ISM (e.g., Fumagalli et al. 2011b; Ribaudo et al. 2011). Indeed, the interplay between galaxies and the LLS is a highly active area of research which includes studies of the so-called circumgalactic medium (CGM; e.g., Steidel et al. 2010; Werk et al. 2013; Prochaska et al. 2014a).

For many decades, LLS have been surveyed in quasar spectra (e.g., Tytler 1982; Sargent et al. 1989; StorrieLombardi et al. 1994), albeit often from heterogeneous samples. These works established the high incidence of LLSs which evolves rapidly with redshift. With the realization of massive spectral data sets, a renaissance of LLS surveys has followed yielding statistically robust measurements from homogenous and well-selected quasar samples (Prochaska et al. 2010; Songaila \& Cowie 2010; Ribaudo et al. 2011; Fumagalli et al. 2013; O'Meara et al. 2013). Analysis of these hundreds of systems reveals an incidence of approximately 1.2 systems per unit redshift at $z \sim 3$ that evolves steeply with redshift $\ell(z) \propto(1+z)^{1.5}$ for $z \approx 1-5$ (Ribaudo et al. 2011; Fumagalli et al. 2013). With these same spectra, researchers have further measured the mean free path of ionizing radiation $\left(\lambda_{\text {mfp }}^{912}\right.$; Prochaska et al. 2009; Fumagalli et al. 2013; O'Meara et al. 2013; Worseck et al. 2014), which sets the intensity and shape of the extragalactic UV background (EUVB). Following the redshift evolution of the LLS incidence, $\lambda_{\mathrm{mfp}}^{912}$ also evolves steeply with the expanding universe, implying a more highly ionized universe with advancing cosmic time (Worseck et al. 2014).

The preponderance of LLSs bespeaks a major reservoir of baryons. In particular, given the apparent paucity of heavy elements within galaxies (e.g., Bouché et al. 2006; Peeples et al. 2014), the LLSs may present the dominant reservoir of metals in the universe (e.g., Prochaska et al. 2006). However, a precise calculation of the heavy elements within LLSs and their contribution to the cosmic budget has not yet been achieved. Despite our success at surveying hundreds of LLSs, there have been few studies resolving their physical properties, and these have generally examined a few individual cases (e.g., Steidel 1990; Prochaska 1999) or composite spectra (Fumagalli et al. 2013). This reflects both the challenges related to data acquisition and analysis together with a historical focus in the community toward the ISM of galaxies (probed by DLAs) and the more diffuse intergalactic medium (IGM).

At $z>2$, a few works have examined the set of LLSs with high $\mathrm{H}_{\mathrm{I}}$ column density $\left(N_{\mathrm{H}} \geqslant 10^{19} \mathrm{~cm}^{-2}\right)$, generally termed the super-LLSs (SLLSs) or sub-damped Ly $\alpha$ systems (subDLAs). Their $N_{\mathrm{H} \text { I }}$ frequency distribution $f\left(N_{\mathrm{H}}, \mathrm{X}\right)$ and chemical abundances have been analyzed from a modestly sized sample (Dessauges-Zavadsky et al. 2003; Péroux et al. 2005; O'Meara et al. 2007; Som et al. 2013; Zafar et al. 2013). Ignoring ionization corrections, which may not be justified, these SLLSs exhibit metallicities of approximately 1/ 10 solar, comparable to the enrichment level of the higher- $N_{\mathrm{H}}$, DLAs; (Rafelski et al. 2012). In addition, a few LLSs have received special attention owing to their peculiar metal- 
Table 1

Journal of Hires Observations

\begin{tabular}{|c|c|c|c|c|c|c|c|c|c|c|}
\hline QSO & Alt. Name & $\begin{array}{l}\text { R.A. } \\
\text { (J2000) }\end{array}$ & $\begin{array}{c}\text { decl. } \\
\text { (J2000) }\end{array}$ & $\begin{array}{l}r / V^{\mathrm{a}} \\
(\mathrm{mag})\end{array}$ & $z_{\mathrm{em}}$ & $\begin{array}{l}\text { Date } \\
\text { (UT) }\end{array}$ & Slit $^{\mathrm{b}}$ & Mode & $\begin{array}{l}\text { Exp } \\
\text { (s) }\end{array}$ & $\begin{array}{c}\mathrm{S} / \mathrm{N}^{\mathrm{c}} \\
\left(\mathrm{pix}^{-1}\right)\end{array}$ \\
\hline SDSS0121+1448 & & $01: 21: 56.03$ & $+14: 48: 23.8$ & 17.1 & 2.87 & 2004 Sep 08 & $\mathrm{C} 1$ & HIRESb & 7200 & $15 / 26$ \\
\hline PSS0133+0400 & & $01: 33: 40.4$ & $+04: 00: 59$ & 18.3 & 4.13 & 2006 Dec 27 & $\mathrm{C} 1$ & HIRESr & 7200 & $14 / 20$ \\
\hline SDSS0157-0106 & & 01:57:41.56 & $-01: 06: 29.6$ & 18.2 & 3.564 & 2003 Dec 18 & $\mathrm{C} 5$ & HIRESr & 9000 & $\mathrm{X} / 14$ \\
\hline Q0201+36 & & 02:04:55.60 & $+36: 49: 18.0$ & 17.5 & 2.912 & 2004 Oct 06 & $\mathrm{C} 1$ & HIRESb & 3600 & $4.5 / 9$ \\
\hline PSS0209+0517 & & 02:09:44.7 & $+05: 17: 14$ & 17.8 & 4.18 & 2007 Sep 18 & $\mathrm{C} 1$ & HIRESr & 11100 & $31 / 24$ \\
\hline \multirow[t]{2}{*}{ Q0207-003 } & & 02:09:51.1 & $-00: 05: 13$ & 17.1 & 2.86 & 2004 Sep 08 & $\mathrm{C} 1$ & HIRESb & 5400 & $15 / 40$ \\
\hline & & & & & & 2004 Sep 09 & $\mathrm{C} 1$ & HIRESb & 8100 & \\
\hline LB0256-0000 & & 02:59:05.6 & $+00: 11: 22$ & 17.7 & 3.37 & 2006 Jan 03 & $\mathrm{C} 5$ & HIRESb & 7049 & $11 / 17$ \\
\hline Q0301-005 & & 03:03:41.0 & $-00: 23: 22$ & 17.6 & 3.23 & 2004 Sep 09 & $\mathrm{C} 1$ & HIRESb & 7800 & $X / 15$ \\
\hline \multirow[t]{2}{*}{ Q0336-01 } & & 03:39:01.0 & $-01: 33: 18$ & 18.2 & 3.20 & 2005 Oct 26 & $\mathrm{C} 5$ & HIRESb & 3600 & $\mathrm{X} / 10$ \\
\hline & & & & & & 2003 Nov 01 & $\mathrm{C} 1$ & HIRESr & 10800 & 15 \\
\hline SDSS0340-0159 & & $03: 40: 24.57$ & $-05: 19: 09.2$ & 17.95 & 2.34 & 2008 Oct 06 & $\mathrm{C} 1$ & HIRESb & 3000 & $7 / 15$ \\
\hline HE0340-2612 & & $03: 42: 27.8$ & $-26: 02: 43$ & 17.4 & 3.14 & 2005 Oct 26 & $\mathrm{C} 1$ & HIRESb & 7200 & $17 / X$ \\
\hline SDSS0731+2854 & & $07: 31: 49.5$ & $+28: 54: 48.6$ & 18.5 & 3.676 & 2006 Jan 04 & $\mathrm{C} 5$ & HIRESb & 7200 & $\mathrm{X} / 15$ \\
\hline \multirow[t]{2}{*}{ Q0731+65 } & & $07: 36: 21.1$ & $+65: 13: 12$ & 18.5 & 3.03 & 2005 Oct 28 & $\mathrm{C} 5$ & HIRESb & 5400 & $\mathrm{X} / 16$ \\
\hline & & & & & & 2006 Jan 04 & $\mathrm{C} 5$ & HIRESb & 7200 & $\mathrm{X} / 12$ \\
\hline \multirow[t]{2}{*}{$\mathrm{J} 0753+4231$} & & 07:53:03.3 & $+42: 31: 30$ & 17.92 & 3.59 & 2005 Oct 26 & $\mathrm{C} 5$ & HIRESb & 3300 & $\mathrm{X} / 12$ \\
\hline & & & & & & 2005 Oct 28 & $\mathrm{C} 5$ & HIRESb & 4800 & $\mathrm{X} / 16$ \\
\hline SDSS0826+3148 & & 08:26:19.7 & $+31: 48: 48$ & 17.76 & 3.093 & 2006 Dec 27 & $\mathrm{C} 1$ & HIRESr & 7900 & $37 / 22$ \\
\hline $\mathrm{J} 0828+0858$ & & $08: 28: 49.2$ & $+08: 58: 55$ & 18.30 & 2.271 & 2012 Apr 14 & $\mathrm{C} 1$ & HIRESb & 1295 & $6 / 9$ \\
\hline J0900+4215 & & 09:00:33.5 & $+42: 15: 46$ & 16.98 & 3.290 & 2005 Apr 15 & $\mathrm{C} 1$ & HIRESb & 4700 & $\mathrm{X} / 20$ \\
\hline J0927+5621 & & 09:27:05.9 & $+56: 21: 14$ & 18.22 & 2.28 & 2005 Apr 14 & $\mathrm{C} 5$ & HIRESb & 8500 & $6 / 20$ \\
\hline J0942+0422 & & $09: 42: 02.0$ & $+04: 22: 44$ & 17.18 & 3.28 & 2005 Mar 18 & $\mathrm{C} 1$ & HIRESb & 7200 & $27 / X$ \\
\hline $\mathrm{J} 0953+5230$ & & 09:53:09.0 & $+52: 30: 30$ & 17.66 & 1.88 & 2005 Mar 18 & $\mathrm{C} 1$ & HIRESb & 7200 & $18 / 22$ \\
\hline \multirow[t]{2}{*}{ Q0956+122 } & & 09:58:52.2 & $+12: 02: 44$ & 17.6 & 3.29 & 2006 Jan 03 & $\mathrm{C} 5$ & HIRESb & 7200 & $\mathrm{X} / 40$ \\
\hline & & & & & & 2006 Apr 07 & $\mathrm{C} 1$ & HIRESr & 1800 & $15 / 10$ \\
\hline \multirow[t]{3}{*}{ HS1011+4315 } & & $10: 14: 47.1$ & $+43: 00: 31$ & 16.1 & 3.1 & 2005 Apr 14 & $\mathrm{C} 5$ & HIRESb & 5100 & $\mathrm{X} / 40$ \\
\hline & & & & & & 2007 Apr 27 & B2 & HIRESr & 3600 & $47 / 47$ \\
\hline & & & & & & 2007 Apr 28 & B2 & HIRESr & 3600 & $47 / 47$ \\
\hline $\mathrm{J} 1019+5246$ & & $10: 19: 39.1$ & $+52: 46: 28$ & 17.92 & 2.170 & 2007 Apr 11 & $\mathrm{C} 1$ & HIRESb & 7200 & $11 / 16$ \\
\hline Q1017+109 & & $10: 20: 10.0$ & $+10: 40: 02$ & 17.5 & 3.15 & 2006 Apr 06 & $\mathrm{C} 5$ & HIRESb & 7200 & $25 / X$ \\
\hline $\mathrm{J} 1035+5440$ & & $10: 35: 14.2$ & $+54: 40: 40$ & 18.21 & 2.988 & 2008 Mar 25 & $\mathrm{C} 1$ & HIERSr & 10800 & $23 / 24$ \\
\hline SDSS1040+5724 & & $10: 40: 18.5$ & $+57: 24: 48$ & 18.30 & 3.409 & 2006 Jan 04 & $\mathrm{C} 5$ & HIRESb & 8100 & $\mathrm{X} / 12$ \\
\hline Q1108-0747 & & $11: 11: 13.6$ & $-08: 04: 02$ & 18.1 & 3.92 & 2006 Apr 07 & $\mathrm{C} 1$ & HIRESr & 7200 & $30 / 10$ \\
\hline $\mathrm{J} 1131+6044$ & & $11: 31: 30.4$ & $+60: 44: 21$ & 17.73 & 2.921 & 2006 Dec 26 & $\mathrm{C} 1$ & HIRESb & 7200 & $14 / 18$ \\
\hline $\mathrm{J} 1134+5742$ & & 11:34:19.0 & $+57: 42: 05$ & 18.20 & 3.522 & 2006 Jan 05 & $\mathrm{C} 5$ & HIRESr & 6300 & $26 / 22$ \\
\hline J1159-0032 & & 11:59:40.7 & $-00: 32: 03$ & 18.10 & 2.034 & 2012 Apr 14 & $\mathrm{C} 1$ & HIRESb & 2400 & $5 / 7$ \\
\hline Q1206+1155 & & 12:09:18.0 & $+09: 54: 27$ & 17.6 & 3.11 & 2006 Apr 06 & $\mathrm{C} 5$ & HIRESb & 7200 & $23 / \mathrm{X}$ \\
\hline Q1330+0108 & & $13: 32: 54.4$ & $+00: 52: 51$ & 18.2 & 3.51 & 2006 Apr 07 & $\mathrm{C} 1$ & HIRESr & 7200 & $11 / 9$ \\
\hline HS1345+2832 & & $13: 48: 11.7$ & $+28: 18: 02$ & 16.8 & 2.97 & 2005 Apr 14 & $\mathrm{C} 5$ & HIRESb & 4800 & $\mathrm{X} / 27$ \\
\hline PKS1354-17 & & $13: 57: 06.07$ & $-17: 44: 01.9$ & 18.5 & 3.15 & 2007 Apr 28 & $\mathrm{C} 5$ & HIRESr & 7200 & $8 / 7$ \\
\hline $\mathrm{J} 1407+6454$ & & $14: 07: 47.2$ & $+64: 54: 19$ & 17.24 & 3.11 & 2005 Apr 14 & $\mathrm{C} 5$ & HIRESb & 5400 & $\mathrm{X} / 20$ \\
\hline HS1431+3144 & & $14: 33: 16.0$ & $+31: 31: 26$ & 17.1 & 2.94 & 2006 Apr 06 & $\mathrm{C} 5$ & HIRESb & 6000 & $25 / 43$ \\
\hline $\mathrm{J} 1454+5114$ & & $14: 54: 08.9$ & $+51: 14: 44$ & 17.59 & 3.644 & 2005 Jul 14 & $\mathrm{C} 5$ & HIRESr & 1800 & $10 / 7.5$ \\
\hline $\mathrm{J} 1509+1113$ & & $15: 09: 32.1$ & $+11: 13: 14$ & 19.0 & 2.11 & 2012 Apr 15 & $\mathrm{C} 1$ & HIRESb & 5200 & $4 / 7$ \\
\hline \multirow[t]{3}{*}{$\mathrm{J} 1555+4800$} & & $15: 55: 56.9$ & $+48: 00: 15$ & 19.1 & 3.297 & 2005 Apr 15 & $\mathrm{C} 5$ & HIRESr & 10800 & $13 / 10$ \\
\hline & & & & & & 2005 Jul 14 & $\mathrm{C} 5$ & HIRESr & 10800 & \\
\hline & & & & & & 2006 Jun 04 & $\mathrm{C} 5$ & HIRESr & 7200 & \\
\hline $\mathrm{J} 1608+0715$ & & $16: 08: 43.9$ & $+07: 15: 09$ & 16.60 & 2.88 & 2007 Apr 11 & $\mathrm{C} 1$ & HIRESb & 9000 & $11 / 26$ \\
\hline \multirow[t]{4}{*}{$\mathrm{J} 1712+5755$} & & $17: 12: 27.74$ & $+57: 55: 06$ & 17.46 & 3.01 & 2004 Sep 09 & $\mathrm{C} 1$ & HIRESb & 3600 & $\mathrm{X} / 12$ \\
\hline & & & & & & 2005 May 02 & $\mathrm{C} 5$ & HIRESb & 3900 & \\
\hline & & & & & & 2006 Aug 19 & $\mathrm{C} 1$ & HIRESb & 3900 & \\
\hline & & & & & & 2006 Aug 20 & $\mathrm{C} 1$ & HIRESb & 3900 & \\
\hline \multirow[t]{2}{*}{$\mathrm{J} 1733+5400$} & & $17: 33: 52.23$ & $+54: 00: 30$ & 17.35 & 3.43 & 2005 May 02 & $\mathrm{C} 5$ & HIRESb & 5400 & $\mathrm{X} / 30$ \\
\hline & & & & & & 2007 Aug 22 & $\mathrm{C} 1$ & HIRESr & 5400 & $35 / 35$ \\
\hline $\mathrm{J} 2123-0050$ & & $21: 23: 29.46$ & $-00: 50: 53$ & 16.43 & 2.26 & 2006 Aug 20 & E3 & HIRESb & 21600 & $30 / 67$ \\
\hline Q2126-1538 & & $21: 29: 12.2$ & $-15: 38: 41$ & 17.3 & 3.27 & 2004 Sep 08 & $\mathrm{C} 1$ & HIRESb & 7200 & $10 / 16$ \\
\hline LB2203-1833 & & $22: 06: 39.6$ & $-18: 18: 46$ & 18.4 & 2.73 & 2004 Sep 09 & $\mathrm{C} 1$ & HIRESb & 5400 & \\
\hline Q2231-00 & LBQS 2231-0015 & $22: 34: 08.8$ & $+00: 00: 02$ & 17.4 & 3.025 & 1995 Nov 01 & $\mathrm{C} 5$ & HIRESO & 14400 & 30 \\
\hline SDSS2303-0939 & & $23: 03: 01.5$ & $-09: 39: 31$ & 17.68 & 3.455 & 2005 Nov 08 & $\mathrm{C} 5$ & HIRESr & 7200 & $25 / 29$ \\
\hline SDSS2315+1456 & & $23: 15: 43.6$ & $+14: 56: 06$ & 18.52 & 3.377 & 2006 Jun 04 & C5 & HIRESr & 4800 & $16 / 11$ \\
\hline
\end{tabular}


Table 1

(Continued)

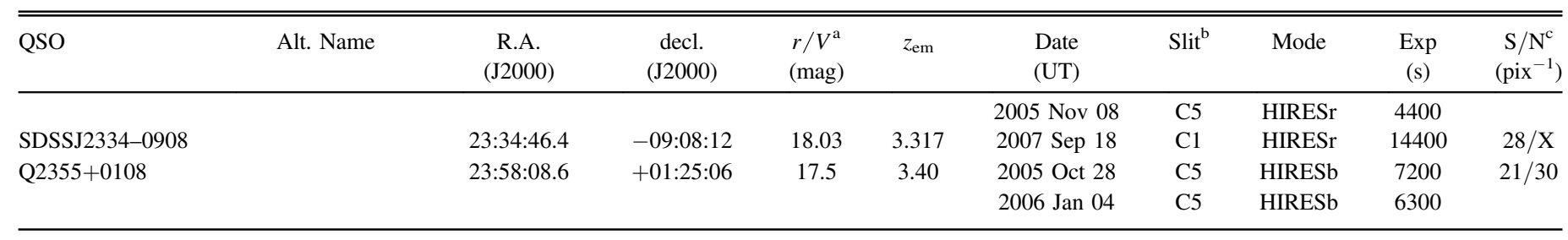

Notes.

${ }^{a}$ Magnitude from the SDSS database ( $r$ band) or as listed in the SIMBAD Astronomical Database $(V$ band).

b Decker employed.

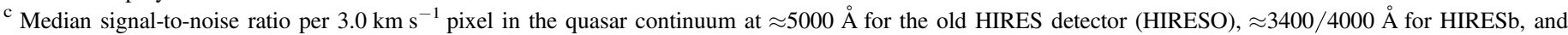
$\approx 6000 / 8000 \AA$ for HIRESr. An "X" indicates no wavelength coverage or that the S/N was compromised by an LLS.

enrichment (Prochaska et al. 2006; Fumagalli et al. 2011a) and/or the detection of $\mathrm{D}$ for studies of Big Bang Nucleosynthesis (e.g., Burles \& Tytler 1998; O'Meara et al. 2006). Most recently, a sample of 15 LLSs has been surveyed for highly ionized $\mathrm{O}_{\mathrm{VI}}$ absorption (Lehner et al. 2014), which is present at a high rate. A comprehensive study of the absorption-line properties of the LLSs at high redshift, however, has not yet been performed.

Scientifically, we have two primary motivations to survey the LLSs at $z>2$. First and foremost, we aim to dissect the physical nature of the gas that dominates the opacity to ionizing radiation in the universe. One suspects that these LLSs trace a diverse set of overdense structures ranging from galactic gas to the densest filaments of the cosmic web. Such diversity may manifest in an wide distribution of observed properties (e.g., metal enrichment, ionization state, kinematics). Second, modern theories of galaxy formation predict that the gas fueling star formation accretes onto galaxies in cool, dense streams (e.g., Kereš et al. 2005; Dekel et al. 2009). Radiative transfer analysis of hydrodynamic simulations of this process predict a relatively high cross-section of optically thick gas around galaxies (e.g., Faucher-Giguère \& Kereš 2011; Fumagalli et al. 2011b, 2014; Faucher-Giguere et al. 2014). Indeed, an optically thick CGM envelops the massive galaxies hosting $z \sim 2$ quasars (Hennawi \& Prochaska 2007; Prochaska et al. 2013), LLSs are observed near Lyman break galaxies (Rudie et al. 2012), and such gas persists around present-day $L^{*}$ galaxies (e.g., Chen et al. 2010; Werk et al. 2013). The latter has inspired, in part, surveys of the LLSs at $z<1$ with ultraviolet spectroscopy (e.g., Ribaudo et al. 2011; Lehner et al. 2013).

Thus motivated, we have obtained a large data set of highdispersion spectroscopy on $z>3$ quasars at the Keck and Las Campanas Observatories. We have supplemented this program with additional spectra obtained to survey the DLAs (e.g., Prochaska et al. 2007; Berg et al. 2014) and the IGM (e.g., Faucher-Giguère et al. 2008). In this paper, we present the comprehensive data set of column density measurements on over 150 LLSs. Future manuscripts will examine the metallicity, chemical abundances, kinematics, and ionization state of this gas. This manuscript is outlined as follows. Section 2 describes the data set analyzed including a summary of the observations and procedures for generating calibrated spectra. We define an LLS in Section 3 and detail the procedures followed to estimate the $\mathrm{H}_{\mathrm{I}}$ column densities in Section 4. Section 5 presents measurements of the ionic column densities and the primary results of an empirical assessment of these data are given in Section 6. A summary in Section 7 concludes the paper.

\section{DATA}

This section describes the steps taken to generate a large data set of high-dispersion, calibrated spectra of high redshift LLSs.

\subsection{Our Survey}

The sample presented in this manuscript is intended to be a nearly all-inclusive set of LLSs discovered in the highdispersion (echelle or echellette; $R>5000$ ) spectra that we have gathered at the Keck and Magellan telescopes. Regarding Keck, we have examined all of the data obtained by Principal Investigators (PIs) A. M. Wolfe and J. X. Prochaska at the W. M. Keck Observatory through 2012 April, and from PIs Burles, O'Meara, Bernstein, and Fumagalli at Magellan through 2012 July. We also include the Keck spectra analyzed by Penprase et al. (2010).

Each spectrum was visually inspected for the presence of damped Ly $\alpha$ absorption and/or a continuum break at wavelengths $\lambda<912 \AA$ in the quasar rest-frame. The complex combination of spectral signal-to-noise ratio $(\mathrm{S} / \mathrm{N})$, wavelength coverage, and quasar emission redshift $z_{\mathrm{em}}$ leads to a varying sensitivity to an LLS. No attempt is made here to define a statistical sample, e.g., to assess the random incidence of LLSs nor their $N_{\mathrm{H}}$ frequency distribution $f\left(N_{\mathrm{H}}, \mathrm{X}\right)$. We refer the reader to previous manuscripts on this topic (Prochaska et al. 2010; Fumagalli et al. 2013). Because our selection is based solely on $\mathrm{H}$ I absorption, however, we believe the sample is largely unbiased with respect to other properties of the gas, e.g., metal-line absorption, kinematics, ionization state.

The sample was limited during the survey by: (1) generally ignoring LLSs with absorption redshifts within $3000 \mathrm{~km} \mathrm{~s}^{-1}$ of the reported quasar redshift $z_{\mathrm{em}}$, so-called proximate LLS or PLLS; and (2) generally ignoring LLSs with $N_{\mathrm{H} \text { I }}<10^{17.3} \mathrm{~cm}^{-2}$, especially when the $\mathrm{S} / \mathrm{N}$ was poor near the Lyman limit. We note further that many of the Keck spectra were obtained to study DLAs at $z>2$ (e.g., Prochaska et al. 2001, 2007; Rafelski et al. 2012; Neeleman et al. 2013; Berg et al. 2014). We have ignored systems targeted as DLAs and also absorbers within $\approx 1500 \mathrm{~km} \mathrm{~s}^{-1}$ of these DLAs because the DLA system complicates analysis of the H I Lyman series and metal-line transitions of any nearby LLS. In Section 3, we offer a strict definition for an LLS to define our sample of 157 systems. 
Table 2

Journal of Mike Observations

\begin{tabular}{|c|c|c|c|c|c|c|c|c|c|c|}
\hline QSO & Alt. Name & $\begin{array}{l}\text { R.A. } \\
\text { (J2000) }\end{array}$ & $\begin{array}{l}\text { decl. } \\
\text { (J2000) }\end{array}$ & $\begin{array}{l}r / V^{\mathrm{a}} \\
(\mathrm{mag})\end{array}$ & $z_{\mathrm{em}}$ & $\begin{array}{l}\text { Date } \\
\text { (UT) }\end{array}$ & $\begin{array}{l}\text { Slit }^{\mathrm{b}} \\
\left({ }^{\prime \prime}\right)\end{array}$ & $\begin{array}{l}\operatorname{Exp} \\
(\mathrm{s})\end{array}$ & $\begin{array}{c}\mathrm{S} / \mathrm{N}_{\text {blue }}{ }^{\mathrm{c}} \\
\left(\mathrm{pix}^{-1}\right)\end{array}$ & $\begin{array}{l}\mathrm{S} / \mathrm{N}_{\mathrm{red}}{ }^{\mathrm{d}} \\
\left(\mathrm{pix}^{-1}\right)\end{array}$ \\
\hline Q0001-2340 & & 00:03:45.0 & $-23: 23: 46$ & 16.7 & 2.262 & 2005 Sep 10 & 1.0 & 3000 & $3 / 27$ & $16 / 19$ \\
\hline \multirow[t]{2}{*}{ J0103-3009 } & LBQS0101-3025 & 01:03:55.3 & $-30: 09: 46$ & 17.6 & 3.15 & 2004 Sep 02 & 1.0 & 2400 & $7 / 9$ & $8 / 10$ \\
\hline & & & & & & 2004 Sep 04 & 1.0 & 2400 & $4 / 7$ & $7 / 8$ \\
\hline SDSSJ0106+0048 & & 01:06:19.2 & $+00: 48: 23.3$ & 19.03 & 4.449 & 2003 Aug 26 & 1.0 & 8000 & $\mathrm{X} / \mathrm{X}$ & $8 / 9$ \\
\hline SDSSJ0124+0044 & & 01:24:03.8 & $+00: 44: 32.7$ & 17.9 & 3.834 & 2003 Aug 28 & 1.0 & 8000 & $\mathrm{X} / 4$ & $24 / 17$ \\
\hline SDSSJ0209-0005 & & 02:09:50.7 & $-00: 05: 06$ & 16.9 & 2.856 & 2005 Sep 10 & 1.0 & 5700 & $\mathrm{X} / 10$ & $11 / 9$ \\
\hline SDSSJ0244-0816 & & 02:44:47.8 & $-08: 16: 06$ & 18.2 & 4.068 & 2003 Aug 26 & 1.0 & 5500 & $X / 2$ & $24 / 12$ \\
\hline \multirow[t]{2}{*}{ HE0340-2612 } & & $03: 42: 27.8$ & $-26: 02: 43$ & 17.4 & 3.14 & 2004 Sep 02 & 1.0 & 2400 & $6 / 11$ & $12 / 19$ \\
\hline & & & & & & 2004 Sep 04 & 1.0 & 2400 & & \\
\hline SDSSJ0344-0653 & & 03:44:02.8 & $-06: 53: 00$ & 18.64 & 3.957 & 2003 Aug 28 & 1.0 & 3000 & $\mathrm{X} / \mathrm{X}$ & $12 / 8$ \\
\hline SDSS0912+0547 & & 09:12:10.35 & $+05: 47: 42$ & 18.05 & 3.248 & 2004 May 10 & 0.7 & 3600 & $2 / 3$ & $6 / X$ \\
\hline SDSSJ0942+0422 & & 09:42:02.0 & $+04: 22: 44$ & 17.18 & 3.28 & 2003 Apr 03 & 0.7 & 6000 & $\mathrm{X} / 9$ & $14 / X X$ \\
\hline HE0940-1050 & & $09: 42: 53.2$ & $-11: 04: 22$ & 16.6 & 3.08 & 2004 May 08 & 1.0 & 7200 & $\mathrm{X} / 40$ & $35 / \mathrm{X}$ \\
\hline \multirow[t]{3}{*}{ SDSSJ0949+0335 } & & $09: 49: 32.3$ & $+03: 35: 31$ & 18.1 & 4.05 & 2003 Apr 04 & 0.7 & 4000 & $4 / 4$ & $12 / 8$ \\
\hline & & & & & & 2003 Apr 05 & 0.7 & 4000 & $3 / 5$ & $11 / 8$ \\
\hline & & & & & & 2003 Apr 06 & 0.7 & 4000 & $2 / 5$ & $11 / 10$ \\
\hline \multirow[t]{2}{*}{ SDSSJ1025+0452 } & & 10:25:09.6 & $+04: 52: 46$ & 18.0 & 3.24 & 2003 Apr 05 & 0.7 & 4000 & $1 / 7$ & $12 / 9$ \\
\hline & & & & & & 2003 Apr 06 & 0.7 & 4000 & $1 / 7$ & $12 / 9$ \\
\hline SDSSJ1028-0046 & & $10: 28: 32.1$ & $-00: 46: 07$ & 17.94 & 2.86 & 2004 May 12 & 1.0 & 6484 & $\mathrm{X} / 8$ & $15 / 6$ \\
\hline SDSSJ1032+0541 & & $10: 32: 49.9$ & $+05: 41: 18.3$ & 17.2 & 2.829 & 2004 May 10 & 1.0 & 7200 & $4 / 13$ & $21 / X$ \\
\hline SDSSJ1034+0358 & & $10: 34: 56.3$ & $+03: 58: 59$ & 17.9 & 3.37 & 2003 Apr 04 & 0.7 & 12000 & $1 / 4$ & $15 / 12$ \\
\hline \multirow[t]{2}{*}{ Q1100-264 } & & $11: 03: 25.6$ & $-26: 45: 06$ & 16.02 & 2.145 & 2005 May 16 & 1.0 & 2000 & $4 / 17$ & $16 / 15$ \\
\hline & & & & & & 2005 May 18 & 1.0 & 2000 & $14 / 37$ & $12 / 9$ \\
\hline HS1104+0452 & & 11:07:08.4 & $+04: 36: 18$ & 17.48 & 2.66 & 2005 May 19 & 1.0 & 2000 & $2 / 9$ & $12 / 14$ \\
\hline SDSSJ1110+0244 & & 11:10:08.6 & $+02: 44: 58$ & 18.3 & 4.12 & 2003 Apr 05 & 0.7 & 8000 & $1 / 3$ & $10 / 7$ \\
\hline SDSSJ1136+0050 & & $11: 36: 21.0$ & $+00: 50: 21$ & 18.1 & 3.43 & 2003 Apr 06 & 0.7 & 8000 & $2 / 7$ & $14 / 13$ \\
\hline SDSSJ1155+0530 & & $11: 55: 38.6$ & $+05: 30: 50$ & 18.1 & 3.47 & 2004 May 10 & 1.0 & 3600 & $3 / 9$ & $12 / 10$ \\
\hline SDSSJ1201+0116 & & $12: 01: 44.4$ & $+01: 16: 11$ & 17.5 & 3.23 & 2003 Apr 03 & 0.7 & 8000 & $1 / 5$ & $15 / 10$ \\
\hline LB1213+0922 & & $12: 15: 39.6$ & $+09: 06: 08$ & 18.26 & 2.723 & 2004 May 13 & 0.7 & 7200 & $3 / 10$ & $10 / 10$ \\
\hline SDSSJ1249-0159 & & $12: 49: 57.2$ & $-01: 59: 28$ & 17.8 & 3.64 & 2003 Apr 06 & 0.7 & 8000 & $1 / 13$ & $16 / 15$ \\
\hline SDSSJ1307+0422 & & $13: 07: 56.7$ & $+04: 22: 15$ & 18.0 & 3.02 & 2004 May 09 & 0.7 & 7200 & $2 / 6$ & $10 / 10$ \\
\hline SDSSJ1337+0128 & & $13: 37: 57.9$ & $+02: 18: 20$ & 18.13 & 3.33 & 2004 May 12 & 1.0 & 6800 & $\mathrm{X} / 10$ & $10 / 9$ \\
\hline SDSSJ1339+0548 & & $13: 39: 42.0$ & $+05: 48: 22$ & 17.8 & 2.98 & 2004 May 10 & 1.0 & 7200 & $6 / 13$ & $14 / 12$ \\
\hline SDSSJ1402+0146 & & $14: 02: 48.1$ & $+01: 46: 34$ & 18.8 & 4.16 & 2003 Apr 05 & 0.7 & 8000 & $1 / 2$ & $12 / 9$ \\
\hline \multirow[t]{2}{*}{ SDSSJ1429-0145 } & Q1426-0131 & $14: 29: 03.0$ & $-01: 45: 18$ & 17.8 & 3.42 & 2003 Apr 06 & 0.7 & 8000 & $2 / 12$ & $13 / 11$ \\
\hline & & & & & & 2005 May 17 & 1.0 & 8000 & & \\
\hline Q1456-1938 & & $14: 56: 50.0$ & $-19: 38: 53$ & 18.7 & 3.16 & 2005 May 18 & 0.7 & 7200 & $5 / 10$ & $14 / 22$ \\
\hline SDSSJ1503+0419 & & $15: 03: 28.9$ & $+04: 19: 49$ & 18.1 & 3.66 & 2004 May 09 & 0.7 & 7200 & $1 / 3$ & $8 / 7$ \\
\hline \multirow[t]{2}{*}{ SDSSJ1558-0031 } & & $15: 58: 10.2$ & $-00: 31: 20$ & 17.6 & 2.83 & 2003 Apr 06 & 0.7 & 6000 & $1 / 6$ & $12 / 8$ \\
\hline & & & & & & 2004 May 10 & 1.0 & 8000 & $3 / 11$ & $18 / 16$ \\
\hline Q1559+0853 & & $16: 02: 22.6$ & $+08: 45: 36.5$ & 17.3 & 2.269 & 2005 May 17 & 1.0 & 4000 & $4 / 17$ & $11 / 17$ \\
\hline \multirow[t]{4}{*}{ SDSSJ1621-0042 } & & $16: 21: 16.9$ & $-00: 42: 50$ & 17.4 & 3.70 & 2003 Apr 03 & 0.7 & 6000 & $\mathrm{X} / 2$ & $11 / 10$ \\
\hline & & & & & & 2003 Apr 05 & 0.7 & 3000 & $\mathrm{X} / 7$ & $16 / 12$ \\
\hline & & & & & & 2003 Apr 06 & 0.7 & 3600 & $\mathrm{X} / 7$ & $18 / 14$ \\
\hline & & & & & & 2004 May 08 & 1.0 & 3600 & $3 / 11$ & \\
\hline PKS2000-330 & Q2000-330 & $20: 03: 24.1$ & $-32: 51: 44$ & 17.3 & 3.77 & 2004 Sep 02 & 1.0 & 4800 & $12 / 35$ & $24 / 19$ \\
\hline B2050-359 & & $20: 53: 44.6$ & $-35: 46: 52$ & 17.7 & 3.49 & 2005 May 18 & 1.0 & 4800 & $\mathrm{X} / 8$ & $10 / 10$ \\
\hline Q2126-1538 & & $21: 29: 12.2$ & $-15: 38: 41$ & 17.3 & 3.27 & 2004 Sep 05 & 1.0 & 4800 & $9 / 25$ & $19 / 23$ \\
\hline \multirow[t]{2}{*}{ HE2215-6206 } & & $22: 18: 51.3$ & $-61: 50: 54$ & 17.5 & 3.32 & 2004 Sep 02 & 1.0 & 2400 & $8 / 20$ & $16 / 14$ \\
\hline & & & & & & 2004 Sep 04 & 1.0 & 4000 & $7 / 17$ & $17 / 19$ \\
\hline SDSSJ2303-0939 & & 23:03:01.4 & $-09: 39: 30$ & 17.68 & 3.455 & 2003 Aug 28 & 1.0 & 8000 & $\mathrm{X} / 14$ & $23 / 20$ \\
\hline HE2314-3405 & & $23: 16: 43.2$ & $-33: 49: 12$ & 16.9 & 2.96 & 2004 Sep 02 & 1.0 & 2400 & $2 / 11$ & $13 / 11$ \\
\hline \multirow[t]{2}{*}{ SDSSJ2346-0016 } & & $23: 46: 25.7$ & $-00: 16: 00$ & 17.77 & 3.49 & 2003 Aug 27 & 1.0 & 8000 & $\mathrm{X} / 14$ & $21 / 26$ \\
\hline & & & & & & 2003 Aug 28 & 1.0 & 3000 & & \\
\hline HE2348-1444 & & $23: 48: 55.4$ & $-14: 44: 37$ & 16.7 & 2.93 & 2004 Sep 02 & 1.0 & 2400 & $14 / 22$ & $30 / 33$ \\
\hline HE2355-5457 & & $23: 58: 33.4$ & $-54: 40: 42$ & 17.1 & 2.94 & 2004 Sep 02 & 1.0 & 2400 & $17 / 7$ & $13 / 15$ \\
\hline
\end{tabular}

Notes.

${ }^{a}$ Magnitude from the SDSS database ( $r$ band) or as listed in the SIMBAD Astronomical Database ( $V$ band).

${ }^{\mathrm{b}}$ Slit width employed. For the blue (red) side, a $1^{\prime \prime}$ slit yields a FWHM resolution of 10.7 (13.6) $\mathrm{km} \mathrm{s}^{-1}$ for a source that fills the slit.

${ }^{\mathrm{c}}$ Median signal-to-noise per $3.0 \mathrm{~km} \mathrm{~s}^{-1}$ pixel in the quasar continuum at $\approx 3400 / 4000 \AA$. An X designates no flux.

${ }^{\mathrm{d}}$ Median signal-to-noise per $4.2 \mathrm{~km} \mathrm{~s}^{-1}$ pixel in the quasar continuum at $\approx 6000 / 8000 \AA$. An X designates no flux. 


\subsection{Observations}

We present data obtained at the W. M. Keck and Las Campanas Observatories using the twin $10 \mathrm{~m}$ Keck I and Keck II telescopes and the twin $6.5 \mathrm{~m}$ Baade and Clay telescopes. Altogether, we used four spectrometers: (1) the High Resolution Echelle Spectrometer (HIRES; Vogt et al. 1994); (2) the Echellette Spectrograph and Imager (ESI; Sheinis et al. 2002); (3) the Magellan Inamori Kyocera Echelle (MIKE; Bernstein et al. 2003); and (4) the Magellan Echellette Spectrograph (MagE; Marshall et al. 2008).

The MagE spectra were presented in Fumagalli et al. (2013) and we refer the reader to that manuscript for details on the observations and data reduction. Similarly the ESI observations have been published previously in a series of papers (Prochaska et al. 2003b, 2007; O’Meara et al. 2007; Rafelski et al. 2012).

Observing logs for the HIRES and MIKE spectra are provided in Tables 1 and 2. A significant fraction of these data have been analyzed previously (e.g., O'Meara et al. 2007; Faucher-Giguère et al. 2008; Neeleman et al. 2013), but not for a comprehensive LLS survey.

\subsection{Data Reduction}

The HIRES spectra were reduced with the HIRedux ${ }^{6}$ software package, primarily as part of the KODIAQ project (Lehner et al. 2014). Briefly, each spectral image was biassubtracted, flat-fielded with pixel flats, and wavelengthcalibrated with corresponding ThAr frames. The echelle orders were traced using a traditional flat-field spectral image. The sky background was subtracted with a b-spline algorithm (e.g., Bochanski et al. 2009), and the quasar flux was further traced and optimally extracted with standard techniques. These spectra were flux normalized with a high-order Legendre polynomial and co-added after weighting by the median $\mathrm{S} / \mathrm{N}$ of each order. This yields an individual, wavelength-calibrated spectrum for each night of observation in the vacuum and heliocentric frame. When possible, we then combined spectra from quasars observed on multiple nights with the same instrument configuration.

Processing of the MIKE spectra used the MIRedux package now bundled within the XIDL software package. ${ }^{7}$ This pipeline uses algorithms similar to HIRedux. The primary difference is that the flux is estimated together with the sky using a set of b-spline models which is demanded by the short $5^{\prime \prime}$ slits employed with MIKE. In addition, these data were fluxed prior to coaddition using the reduced spectrum of a spectrophotometric standard (taken from the same night in most cases). Therefore, we provide both fluxed and normalized spectra from this instrument.

Details on the data reduction of ESI and MagE spectra are provided in previous publications (Prochaska et al. 2003b; Fumagalli et al. 2013).

All of the reduced and calibrated spectra are available on the project's website. ${ }^{8}$ The Keck/HIRES spectra will also be provided in the first data release of the KODIAQ project (O’Meara et al. 2015).

\footnotetext{
6 http://www.ucolick.org/ xavier/HIRedux/index.html

7 http://www.ucolick.org/ xavier/IDL/index.html

8 http://www.ucolick.org/ xavier/HD-LLS/DR1
}

\section{LLS DEFINITION}

Before proceeding to our analysis of the sample, we strictly define the LLS. There are three aspects to the definition.

1. The velocity interval analyzed, which also corresponds to a finite redshift window.

2. The $N_{\mathrm{H} \text { I }}$ value of the system.

3. The spatial proximity of the LLS to other astrophysical objects (e.g., the background quasar or a foreground DLA).

Of these three, the first has received the least attention by the community yet may be the most important. Establishing a precise definition, however, is largely arbitrary despite the fact that it may significantly impact the studies that follow. This includes the assessment of gas kinematics (Prochaska \& Wolfe 1997), metallicity (Prochter et al. 2010; Fumagalli et al. 2011a), and even the contribution of LLSs to the cosmic mean free path (Prochaska et al. 2014b). In this paper (and future publications), we adopt an observationally motivated velocity interval of $\pm 500 \mathrm{~km} \mathrm{~s}^{-1}$ centered on the peak optical depth $z_{\mathrm{LLS}}^{\text {peak }}$ of the H I Lyman series. Frequently, we estimate $z_{\text {LLS }}^{\text {peak }}$ from the peak optical depth of a low-ion, metal-line transition. An LLS, then, is all of the optically thick gas at $|\delta v|<500 \mathrm{~km} \mathrm{~s}^{-1}$ from $z_{\mathrm{LLS}}^{\text {peak }}$. In practice, we have not simply summed the $\mathrm{H}_{\mathrm{I}}$ column densities of all $\operatorname{Ly} \alpha$ absorbers within this interval. Instead, we have adopted the integrated $N_{\mathrm{H}}$ estimate from the Lyman limit decrement or adopt $N_{\mathrm{H}}$ from the analysis of damping in the Ly $\alpha$ profiles (see the next section for more detail). As an example of the latter, we treat the two absorbers at $z=3.1878$ and $z=3.1917$ toward PKS2000-330 (Prochter et al. 2010) as a single LLS. Similarly, we sum metal-line absorption identified within the interval although it rarely is detected in intervals that exceeds $200 \mathrm{~km} \mathrm{~s}^{-1}$. Moreover, this window was adjusted further to exclude absorption from unrelated (e.g., higher or lower redshift) systems. While this is an observationally driven definition, we note that it should also capture even the largest peculiar motions within dark matter halos at $z \sim 2$

With $|\delta v|<500 \mathrm{~km} \mathrm{~s}^{-1}$ as the first criterion, we define an LLS as any combination of systems with $N_{\mathrm{H}} \geqslant 10^{17.3} \mathrm{~cm}^{-2}$ within that interval; this yields an integrated optical depth at the Lyman limit $\tau_{912} \geqslant 1$. In practice, we distinguish the LLS from DLAs by requiring that $N_{\mathrm{H}}<10^{20.3} \mathrm{~cm}^{-2}$. Systems with $10^{16}<N_{\mathrm{H}}<10^{17.3} \mathrm{~cm}^{-2}$ are referred to as partial LLSs or pLLSs, and are excluded from analysis in this manuscript. Last, we refer to an LLS within $3000 \mathrm{~km} \mathrm{~s}^{-1}$ of the background quasar as a proximate LLS or PLLS (Prochaska et al. 2008b). There are five PLLSs within our sample satisfying this definition, all with velocity separations of at least $2000 \mathrm{~km} \mathrm{~s}^{-1}$ from the reported quasar redshifts. Altogether, we present measurements for 157 LLSs at redshifts $z_{\text {LLS }}=1.76-4.39$ and with $N_{\mathrm{H}}=10^{17.3}-10^{20.25} \mathrm{~cm}^{-2}$. Here and in future papers we refer to this data set as the highdispersion LLS sample (HD-LLS Sample). We will augment this sample in the years to follow via our web site.

\section{4. $N_{\mathrm{H}}$ ANALYSIS}

Although the continuum opacity of the Lyman limit generates an unambiguous signature in a quasar spectrum, it is generally challenging to precisely estimate the $\mathrm{H}_{\text {I }}$ column density $N_{\mathrm{H} \text { I }}$ for a given LLS system. This follows simply from 
the fact that $\exp \left(-\tau_{912}\right) \ll 1$ for $N_{\mathrm{H} \mathrm{I}}>10^{18} \mathrm{~cm}^{-2}$ and all of the $\mathrm{H}_{\text {I }}$ Lyman series lines are on the saturated portion of the curve of growth for $N_{\mathrm{HI}}<10^{19} \mathrm{~cm}^{-2}$. Furthermore, the damping of Ly $\alpha$ is difficult to measure for $N_{\mathrm{H}} \ll 10^{20} \mathrm{~cm}^{-2}$, especially in low $\mathrm{S} / \mathrm{N}$ spectra or at $z>3$ where IGM blending is substantial.

Our approach to identifying LLS and estimating their $N_{\mathrm{H}}$ values involved two relatively distinct procedures. For LLS with large $N_{\mathrm{H} \text { I }}$ values $\left(>10^{19} \mathrm{~cm}^{-2}\right)$, we searched each spectrum for absorption features with large equivalent widths $W_{\lambda}$ characteristic of a damped Ly $\alpha$ line (i.e., $W_{\lambda} \gg 1 \AA$ ). We then considered whether these candidates could be related to a broad absorption line (BAL) system associated to the background quasar or $\operatorname{Ly} \beta$ associated to a higher redshift DLA. Any such coincidences were eliminated. For the remaining candidates, we performed an analysis of the Ly $\alpha$ profile by overplotting a series of Voigt profiles with $N_{\mathrm{H} \text { I }}>10^{18.5} \mathrm{~cm}^{-2}$, adjusting the local continuum by-eye as warranted. When low-ion metal absorption was detected near the approximate centroid of $\mathrm{Ly} \alpha$, we centered the model to its peak optical depth and refined the $N_{\mathrm{H}}$ value accordingly. We did not, however, require the positive detection of metal-line absorption. In all cases, the Doppler parameter of the model Ly $\alpha$ line was set to $30 \mathrm{~km} \mathrm{~s}^{-1}$.

For cases were the $\mathrm{S} / \mathrm{N}$ was deemed sufficient and lineblending not too severe, we estimated (by visual inspection) a "best" $N_{\mathrm{H} \text { I }}$ value and corresponding $1 \sigma$ uncertainties. Although this procedure is rife with human interaction, we maintain that it offers the most robust assessment (to date) for $N_{\mathrm{H} \text { I }}$ estimation. This is because the dominant uncertainties are systematic (e.g., continuum placement and line-blending), which are difficult to estimate statistically. Figure 1 shows three examples of LLSs with damped Ly $\alpha$ lines giving precisely estimated $N_{\mathrm{H} \text { I }}$ values. Such systems are commonly referred to as SLLSs or sub-DLAs.

We provide the adopted $N_{\mathrm{H} \text { I }}$ values and error estimates of our SLLS sample ( 99 systems with $N_{\mathrm{H}} \geqslant 10^{19} \mathrm{~cm}^{-2}$ ) in Table 3. These represent roughly $2 / 3$ of the total HD-LLS Sample. This high fraction occurs because we only require coverage of $\mathrm{H}_{\text {I }} \mathrm{Ly} \alpha$ to identify and analyze these SLLS. This implies a much larger survey path-length than for the lower $N_{\mathrm{H} \text { I }}$ LLS. In addition, one may identify and analyze multiple SLLSs along a given sightline whereas one is restricted to a single LLS when the Lyman Limit is central to the analysis. Because these SLLSs tend to span nearly the entire $\pm 500 \mathrm{~km} \mathrm{~s}^{-1}$ window that defines an LLS, it is possible that there is additional, optically thick gas not included in our $N_{\mathrm{H}}$ estimate. This will be rare, however, and the underestimate of $N_{\mathrm{H} \text { I }}$ should generally be much less than $10 \%$.

In parallel with the search for LLSs having strong Ly $\alpha$ lines, we inspected each spectrum for a Lyman limit break. For those LLSs that exhibit non-negligible flux at the Lyman limit, i.e., $\tau_{912}<3$, a precise $N_{\mathrm{H} \mathrm{I}}$ estimation may be recovered. In practice, such analysis is hampered by poor sky subtraction and associated IGM absorption that stochastically reduces the quasar flux through the spectral region near the Lyman limit and affects continuum placement. In the following, we have been conservative regarding the systems with $N_{\mathrm{HI}}$ measurements from the Lyman limit flux decrement. We are currently acquiring additional, low-dispersion spectra to confirm the flux at the Lyman limit for a set of the HD-LLS Sample. Figure 2 shows one example of a pLLS observed with both the MIKE and MagE spectrometers. The flux decrement is obvious and one also appreciates the value of higher spectral resolution (with high $\mathrm{S} / \mathrm{N}$ ) for resolving IGM absorption.

For the remainder of the systems identified on the basis of a Lyman limit break, we adopt conservative bounds (i.e., upper and lower limits) to the $N_{\mathrm{H} \text { I }}$ values. These are based primarily on analysis of the Ly $\alpha$ line and the flux at the Lyman limit. The absence of strong damping in the former provides a strict upper limit to $N_{\mathrm{H} \text { I }}$ while the latter sets a firm lower limit. These bounds are provided in Table 3, and Figure 3 shows two examples of these "ambiguous" cases. In practice, the bounds are often an order of magnitude apart, e.g., $10^{17.7} \mathrm{~cm}^{-2}<N_{\mathrm{H}}<10^{18.9} \mathrm{~cm}^{-2}$. Furthermore, it is difficult to estimate the probability distribution function (PDF) of $N_{\mathrm{H} \text { I }}$ within these bounds. One should not, for example, assume a Gaussian PDF centered within the bounds with a dispersion of half the interval. In fact, we expect that the PDF is much closer to uniform, i.e., equal probability for any $N_{\mathrm{H} \text { I }}$ value within the bounds. This expectation is motivated by current estimations of the $N_{\mathrm{HI}}$ frequency distribution $f\left(N_{\mathrm{H}}, \mathrm{X}\right)$ which argue for a uniform distribution of $N_{\mathrm{H}}$ values for randomly selected systems with $N_{\mathrm{HI}} \approx 10^{18} \mathrm{~cm}^{-2}$ (Prochaska et al. 2010; O'Meara et al. 2013). Going forward, we advocate adopting a uniform PDF.

As a cross-check on the analysis, 50 of the sightlines were re-analyzed by a second author to identify LLSs and estimate their $N_{\mathrm{H} \text { I }}$ values. With two exceptions, the values between the two authors agree within the estimated uncertainty and we identify no obvious systematic bias. ${ }^{9}$ These two exceptions have $>0.5$ dex difference due to differing definitions used by the two authors and we have adopted the values corresponding to the strict definition provided in Section 3. This exercise confirms that the uncertainties are dominated by systematic effects, not $\mathrm{S} / \mathrm{N}$ nor the analysis procedures.

Table 3 lists the adopted $N_{\mathrm{H}}$ value, errors on this value, the bounds on $N_{\mathrm{HI}}$, and a flag indicating whether one would assume a normal or uniform PDF. Figure 4 shows a histogram of the adopted $N_{\mathrm{HI}}$ values and a scatter plot against $z_{\mathrm{LLS}}$. It is evident that the HD-LLS Sample is weighted toward higher $N_{\mathrm{H} \text { I }}$ values and $z \sim 3$.

\section{IONIC COLUMN DENSITIES}

For each of the HD-LLS Sample, we inspected the spectra for associated metal-line absorption. Emphasis was placed on transitions with observed wavelengths redward of the Ly $\alpha$ forest. A velocity interval was estimated for the column density measurements based on the cohort of transitions detected. Velocity plots were generated and inspected to search for lineblending. Severely blended lines were eliminated from analysis and intermediate/weak cases were measured but reported as upper limits. All of these assignments were vetted by JXP, MF, and JMO. Figures 5 and 6 show the Si II 1526/Si IV 1393 transitions for three representative SLLSs and the C II 1334/ C IV 1548 transitions for three LLSs with $N_{\mathrm{H}} \lesssim 10^{17.7} \mathrm{~cm}^{-2}$. These data indicate a great diversity of line-strengths for these transitions within the SLLS sample. We also conclude that

\footnotetext{
In fact the formal reduced $\chi^{2}$ for the comparison is significantly less than unity, but this is because the estimated uncertainties include systematic error and because each author analyzed the same data.
} 

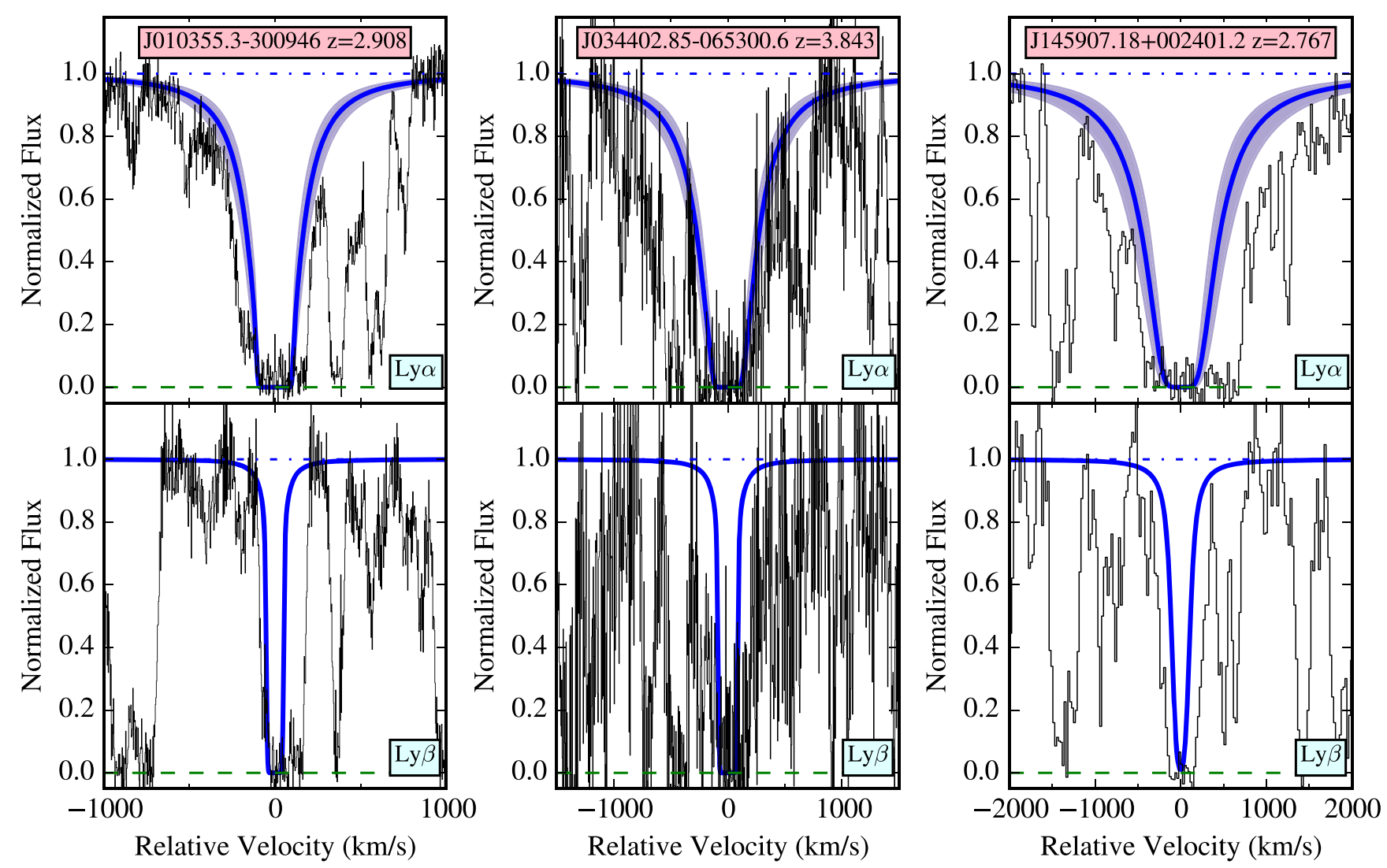

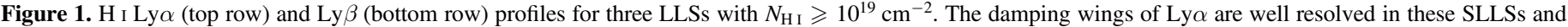
the blue curves indicate for the best-estimates and uncertainty of the $N_{\mathrm{H} \text { I }}$ values. These are well constrained, even in poor $\mathrm{S} / \mathrm{N}$ data.

metal-absorption is dominated by high-ions in the lower $N_{\mathrm{H} \text { I }}$ systems.

Column densities were measured using the apparent optical depth method; (Savage \& Sembach 1991) which gives accurate results for unsaturated line profiles. On the latter point, the echelle data (MIKE, HIRES) have sufficiently high resolution to directly assess line-saturation, i.e., only profiles with minimum normalized flux $f_{\min }$ less than 0.1 may be saturated. For the echellette data (MagE, ESI), however, line-saturation is a concern (e.g., Prochaska et al. 2003b). In general, we have proceeded conservatively by treating most lines as saturated when $f_{\min }<0.5$. For many of the ions analyzed in these LLSs, we observe multiple transitions with differing oscillator strengths and have further assessed line-saturation from the cohort of measurements.

Uncertainties were estimated from a standard propagation of error, which does not include error from continuum placement. To be conservative, we adopt a minimum uncertainty of 0.05 dex to the measurements from a given transition. When multiple transitions from the same ion were measured (e.g. Si II 1304 and $\mathrm{Si}$ II 1526 for $\mathrm{Si}^{+}$), we calculate the ionic column density from the weighted mean. Otherwise, we adopt the measurement from the single transition or a limit from the cohort emphasizing positive detections.

A complete set of tables and figures for the metal-line transitions analyzed for each LLS are given online. Tables 4 and 5 summarize the results for $\mathrm{Al}^{+}, \mathrm{Al}^{++}, \mathrm{Fe}^{+}, \mathrm{C}^{+}, \mathrm{C}^{+3}, \mathrm{O}^{0}$, $\mathrm{Si}^{+}$, and $\mathrm{Si}^{+3}$. A listing of all the measurements from this manuscript is provided in the appendix. Figures 7 and 8 show the column density distributions for a set of $\mathrm{Al}, \mathrm{Fe}, \mathrm{Si}, \mathrm{C}$, and

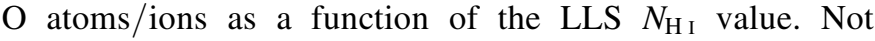
surprisingly, the lower ionization states show an obvious correlation $^{10}$ with $\mathrm{H}$ i column density although there is a large scatter at all values. The near absence of positive detections for $\mathrm{O}_{\text {I }}$ (i.e., $N\left(\mathrm{O}^{0}\right)<10^{14} \mathrm{~cm}^{-2}$ ) at low $N_{\mathrm{H}}$ is also notable. This suggests a rarity of high metallicity gas in systems with $\tau_{912}<10$. The high ions are also positively correlated with the neutral column but with yet larger scatter and much smaller correlation coefficients.

\section{RESULTS}

In the following, we present a set of results derived from the column density measurements of the previous sections. For this manuscript, we restrict the analysis to an empirical investigation. Future studies will introduce additional models and analysis (e.g., photoionization modeling) to interpret the data. We further restrict the discussion to ionic abundances and defer the analysis of kinematics to future work.

\subsection{Ionization State}

As noted above, a full treatment of the ionization state of the gas including the comparison to models will be presented in a future manuscript. We may, however, explore the state of the gas empirically through the examination of ionic ratios that are sensitive to the ionization state of the gas. Figure 9 presents four such ionic ratios against $N_{\mathrm{HI}}$. These primarily compare

\footnotetext{
${ }^{10}$ Taking limits as values, all of these ions have a Spearman rank test probability of less than 0.0001 .
} 
Table 3

$N_{\mathrm{H} \text { I }}$ Estimates for the HD-LLS Sample

\begin{tabular}{|c|c|c|c|c|c|}
\hline Quasar & $z_{\text {peak }}{ }^{a}$ & $N_{\mathrm{HI}}^{\text {low }}$ & $N_{\mathrm{HI}}^{\text {high }}$ & $N_{\mathrm{HI}}^{\text {adopt }}$ & $\mathrm{flg}_{\mathrm{H} \mathrm{I}}$ \\
\hline $\mathrm{J} 1608+0715$ & 1.7626 & 19.10 & 19.70 & $19.40_{-0.30}^{+0.30}$ & 1 \\
\hline $\mathrm{J} 0953+5230$ & 1.7678 & 20.00 & 20.20 & $20.10_{-0.10}^{+0.10}$ & 1 \\
\hline J0927+5621 & 1.7749 & 18.90 & 19.10 & $19.00_{-0.10}^{+0.10}$ & 1 \\
\hline $\mathrm{J} 1509+1113$ & 1.8210 & 18.00 & 19.00 & $18.50_{-0.50}^{+0.50}$ & 2 \\
\hline J101939.15+524627 & 1.8339 & 18.80 & 19.40 & $19.10_{-0.30}^{+0.30}$ & 1 \\
\hline Q1100-264 & 1.8389 & 19.25 & 19.55 & $19.40_{-0.15}^{+0.15}$ & 1 \\
\hline J1159-0032 & 1.9044 & 19.90 & 20.20 & $20.05_{-0.15}^{+0.15}$ & 1 \\
\hline Q0201+36 & 1.9548 & 19.90 & 20.30 & $20.10_{-0.20}^{+0.20}$ & 1 \\
\hline $\mathrm{J} 0828+0858$ & 2.0438 & 19.80 & 20.00 & $19.90_{-0.10}^{+0.10}$ & 1 \\
\hline J2123-0050 & 2.0593 & 19.10 & 19.40 & $19.25_{-0.15}^{+0.15}$ & 1 \\
\hline Q1456-1938 & 2.1701 & 19.55 & 19.95 & $19.75_{-0.20}^{+0.20}$ & 1 \\
\hline J034024.57- 051909 & 2.1736 & 19.15 & 19.55 & $19.35_{-0.20}^{+0.20}$ & 1 \\
\hline Q0001-2340 & 2.1871 & 19.50 & 19.80 & $19.65_{-0.15}^{+0.15}$ & 1 \\
\hline SDSS $1307+0422$ & 2.2499 & 19.85 & 20.15 & $20.00_{-0.15}^{+0.15}$ & 1 \\
\hline $\mathrm{J} 1712+5755$ & 2.3148 & 20.05 & 20.35 & $20.20_{-0.15}^{+0.15}$ & 1 \\
\hline Q2053-3546 & 2.3320 & 18.75 & 19.25 & $19.00_{-0.25}^{+0.25}$ & 1 \\
\hline Q2053-3546 & 2.3502 & 19.35 & 19.85 & $19.60_{-0.25}^{+0.25}$ & 1 \\
\hline Q1456-1938 & 2.3512 & 19.35 & 19.75 & $19.55_{-0.20}^{+0.20}$ & 1 \\
\hline $\mathrm{J} 1131+6044$ & 2.3620 & 19.90 & 20.20 & $20.05_{-0.15}^{+0.15}$ & 1 \\
\hline Q1206+1155 & 2.3630 & 20.05 & 20.45 & $20.25_{-0.20}^{+0.20}$ & 1 \\
\hline HE2314-3405 & 2.3860 & 18.80 & 19.20 & $19.00_{-0.20}^{+0.20}$ & 1 \\
\hline Q0301-005 & 2.4432 & 19.75 & 20.05 & $19.90_{-0.15}^{+0.15}$ & 1 \\
\hline HS1345+2832 & 2.4477 & 19.70 & 20.00 & $19.85_{-0.15}^{+0.15}$ & 1 \\
\hline $\mathrm{J} 1035+5440$ & 2.4570 & 19.40 & 19.90 & $19.65_{-0.25}^{+0.25}$ & 1 \\
\hline Q1337+11 & 2.5080 & 20.00 & 20.30 & $20.15_{-0.15}^{+0.15}$ & 1 \\
\hline SDSS0912+0547 & 2.5220 & 19.15 & 19.55 & $19.35_{-0.20}^{+0.20}$ & 1 \\
\hline SDSS0209-0005 & 2.5228 & 18.90 & 19.20 & $19.05_{-0.15}^{+0.15}$ & 1 \\
\hline LB1213+0922 & 2.5230 & 20.00 & 20.40 & $20.20_{-0.20}^{+0.20}$ & 1 \\
\hline Q0207-003 & 2.5231 & 18.80 & 19.20 & $19.00_{-0.20}^{+0.20}$ & 1 \\
\hline Q0207-003 & 2.5466 & 17.60 & 18.60 & $18.10_{-0.50}^{+0.50}$ & 2 \\
\hline HS1104+0452 & 2.6014 & 19.70 & 20.10 & $19.90_{-0.20}^{+0.20}$ & 1 \\
\hline $\mathrm{J} 2234+0057$ & 2.6040 & 19.25 & 19.75 & $19.50_{-0.25}^{+0.25}$ & 1 \\
\hline $\mathrm{J} 115659.59+551308.1$ & 2.6159 & 18.80 & 19.30 & $19.10_{-0.30}^{+0.30}$ & 1 \\
\hline SDSS1558-0031 & 2.6300 & 19.40 & 19.75 & $19.60_{-0.20}^{+0.20}$ & 1 \\
\hline SDSS0157-0106 & 2.6313 & 19.25 & 19.65 & $19.45_{-0.20}^{+0.20}$ & 1 \\
\hline Q2126-158 & 2.6380 & 19.10 & 19.40 & $19.25_{-0.15}^{+0.15}$ & 1 \\
\hline $\mathrm{Q} 1455+123$ & 2.6481 & 17.30 & 19.40 & $18.35_{-1.05}^{+1.05}$ & 2 \\
\hline LBQS2231-0015 & 2.6520 & 18.70 & 19.30 & $19.10_{-0.40}^{+0.20}$ & 1 \\
\hline SDSS0121+1448 & 2.6623 & 19.05 & 19.40 & $19.25_{-0.20}^{+0.15}$ & 1 \\
\hline SDSSJ0915+0549 & 2.6631 & 17.50 & 18.90 & $18.20_{-0.70}^{+0.70}$ & 2 \\
\hline SDSSJ2319-1040 & 2.6750 & 19.30 & 19.60 & $19.45_{-0.15}^{+0.15}$ & 1 \\
\hline Q0201+36 & 2.6900 & 17.50 & 18.80 & $18.50_{-1.00}^{+0.30}$ & 2 \\
\hline LB2203-1833 & 2.6981 & 19.85 & 20.15 & $20.00_{-0.15}^{+0.15}$ & 1 \\
\hline SDSSJ1551+0908 & 2.7000 & 17.30 & 17.70 & $17.50_{-0.20}^{+0.20}$ & 1 \\
\hline HS1200+1539 & 2.7080 & 17.60 & 18.90 & $18.30_{-0.70}^{+0.70}$ & 2 \\
\hline Q1508+087 & 2.7219 & 19.00 & 19.80 & $19.40_{-0.40}^{+0.40}$ & 1 \\
\hline PMNJ1837-5848 & 2.7289 & 17.50 & 18.70 & $18.10_{-0.60}^{+0.60}$ & 2 \\
\hline FJ2129+00 & 2.7349 & 19.90 & 20.30 & $20.10_{-0.20}^{+0.20}$ & 1 \\
\hline CTS0220 & 2.7390 & 17.30 & 18.70 & $18.00_{-0.70}^{+0.70}$ & 2 \\
\hline SDSS1004+0018 & 2.7460 & 19.60 & 20.00 & $19.80_{-0.20}^{+0.20}$ & 1 \\
\hline SDSSJ0338-0005 & 2.7460 & 19.80 & 20.20 & $20.00_{-0.20}^{+0.20}$ & 1 \\
\hline SDSS1307+0422 & 2.7493 & 17.60 & 18.80 & $18.20_{-0.60}^{+0.60}$ & 2 \\
\hline SDSS1032+0541 & 2.7609 & 17.40 & 17.90 & $17.60_{-0.20}^{+0.20}$ & 1 \\
\hline SDSSJ1459+0024 & 2.7670 & 19.80 & 20.25 & $20.00_{-0.20}^{+0.20}$ & 1 \\
\hline Q2126-158 & 2.7686 & 19.00 & 19.40 & $19.20_{-0.20}^{+0.20}$ & 1 \\
\hline $\mathrm{J} 155738.39+232057$ & 2.7731 & 19.00 & 19.70 & $19.40_{-0.40}^{+0.40}$ & 1 \\
\hline HE2348-1444 & 2.7751 & 17.30 & 17.70 & $17.50_{-0.20}^{+0.20}$ & 1 \\
\hline $\mathrm{J} 1733+5400$ & 2.7790 & 19.50 & 19.90 & $19.70_{-0.20}^{+0.20}$ & 1 \\
\hline
\end{tabular}

Table 3

(Continued)

\begin{tabular}{|c|c|c|c|c|c|}
\hline Quasar & $z_{\text {peak }}{ }^{a}$ & $N_{\mathrm{H} \mathrm{I}}^{\text {low }}$ & $N_{\mathrm{HI}}^{\text {high }}$ & $N_{\mathrm{HI}}^{\text {adopt }}$ & $\mathrm{flg}_{\mathrm{H}}$ \\
\hline CTS0648 & 2.7960 & 17.50 & 18.70 & $18.10_{-0.60}^{+0.60}$ & 2 \\
\hline J095542.12+411655 & 2.8119 & 19.75 & 20.05 & $19.90_{-0.15}^{+0.15}$ & 1 \\
\hline Q0038-4041 & 2.8161 & 17.40 & 17.70 & $17.55_{-0.15}^{+0.15}$ & 1 \\
\hline HE2348-1444 & 2.8187 & 17.10 & 17.40 & $17.25_{-0.15}^{+0.15}$ & 1 \\
\hline J1028-0046 & 2.8239 & 17.70 & 18.30 & $18.00_{-0.30}^{+0.30}$ & \\
\hline SDSSJ0859+0205 & 2.8451 & 17.30 & 18.50 & $17.55_{-0.25}^{+0.95}$ & \\
\hline $\mathrm{J} 1035+5440$ & 2.8460 & 19.55 & 19.85 & $19.70_{-0.15}^{+0.15}$ & 1 \\
\hline $\mathrm{J} 1712+5755$ & 2.8487 & 17.60 & 18.60 & $17.80_{-0.20}^{+0.80}$ & 2 \\
\hline SDSS1034+0358 & 2.8487 & 19.40 & 19.80 & $19.60_{-0.20}^{+0.20}$ & 1 \\
\hline SDSS0826+3148 & 2.8560 & 19.20 & 19.60 & $19.40_{-0.20}^{+0.20}$ & 1 \\
\hline J0239-0038 & 2.8680 & 18.35 & 19.25 & $18.80_{-0.45}^{+0.45}$ & 2 \\
\hline SDSSJ1015+1118 & 2.8699 & 17.50 & 18.85 & $18.20_{-0.70}^{+0.70}$ & 2 \\
\hline LBQS1345-0120 & 2.8831 & 17.90 & 19.30 & $18.60_{-0.70}^{+0.70}$ & 2 \\
\hline CTS0358 & 2.8929 & 19.85 & 20.15 & $20.00_{-0.15}^{+0.15}$ & 1 \\
\hline HE2355-5457 & 2.8949 & 17.20 & 17.60 & $17.40_{-0.20}^{+0.20}$ & 1 \\
\hline Q0101-304 & 2.9082 & 18.95 & 19.25 & $19.10_{-0.15}^{+0.15}$ & 1 \\
\hline $\mathrm{Q} 0731+653$ & 2.9087 & 17.60 & 19.00 & $18.50_{-0.90}^{+0.50}$ & 2 \\
\hline $\mathrm{J} 133146.21+483826$ & 2.9100 & 19.30 & 20.00 & $19.75_{-0.45}^{+0.25}$ & 2 \\
\hline FBQS2129+0037 & 2.9170 & 17.70 & 18.50 & $18.10_{-0.40}^{+0.40}$ & 1 \\
\hline HE0940- 1050 & 2.9170 & 17.35 & 17.65 & $17.50_{-0.15}^{+0.15}$ & 1 \\
\hline SDSS1257-0111 & 2.9180 & 19.75 & 20.15 & $19.95_{-0.20}^{+0.20}$ & 1 \\
\hline UM669 & 2.9269 & 19.80 & 20.20 & $20.00_{-0.20}^{+0.20}$ & 1 \\
\hline J075155.10+451619 & 2.9271 & 19.60 & 20.00 & $19.80_{-0.20}^{+0.20}$ & 1 \\
\hline SDSSJ0931-0000 & 2.9271 & 19.00 & 19.50 & $19.25_{-0.25}^{+0.25}$ & 1 \\
\hline UM184 & 2.9301 & 17.40 & 18.90 & $18.50_{-1.10}^{+0.40}$ & 2 \\
\hline $\mathrm{J} 140747.23+645319$ & 2.9346 & 20.00 & 20.40 & $20.20_{-0.20}^{+0.20}$ & 1 \\
\hline Q0301-005 & 2.9408 & 18.30 & 18.90 & $18.70_{-0.40}^{+0.20}$ & 1 \\
\hline SDSS2315+1456 & 2.9430 & 18.50 & 19.40 & $18.80_{-0.30}^{+0.30}$ & 1 \\
\hline SDSS0127-00 & 2.9440 & 19.60 & 20.00 & $19.80_{-0.20}^{+0.20}$ & 1 \\
\hline SDSS1339+0548 & 2.9520 & 17.40 & 17.90 & $17.65_{-0.25}^{+0.25}$ & 1 \\
\hline Q2126-158 & 2.9676 & 17.10 & 17.50 & $17.30_{-0.20}^{+0.20}$ & 1 \\
\hline SDSSJ1550+0537 & 2.9801 & 19.50 & 19.95 & $19.75_{-0.25}^{+0.25}$ & 1 \\
\hline J140243.97+590958 & 2.9859 & 19.00 & 19.50 & $19.30_{-0.30}^{+0.30}$ & 1 \\
\hline SDSSJ1615+0608 & 2.9879 & 18.50 & 19.50 & $19.20_{-0.70}^{+0.30}$ & 2 \\
\hline Q2053-3546 & 2.9890 & 19.95 & 20.25 & $20.10_{-0.15}^{+0.15}$ & 1 \\
\hline SDSSJ0148-0907 & 2.9951 & 17.40 & 17.90 & $17.55_{-0.15}^{+0.15}$ & 1 \\
\hline H0449-1325 & 2.9980 & 17.40 & 17.70 & $17.55_{-0.15}^{+0.15}$ & 1 \\
\hline Q1456-1938 & 3.0009 & 16.95 & 17.35 & $17.15_{-0.20}^{+0.20}$ & 1 \\
\hline SDSS1034+0358 & 3.0026 & 18.95 & 19.25 & $19.10_{-0.15}^{+0.15}$ & 1 \\
\hline J0814+5029 & 3.0040 & 19.60 & 19.90 & $19.75_{-0.15}^{+0.15}$ & 1 \\
\hline PKS1354- 17 & 3.0069 & 19.15 & 19.65 & $19.40_{-0.25}^{+0.25}$ & 1 \\
\hline HE0340-2612 & 3.0121 & 17.80 & 18.40 & $18.10_{-0.30}^{+0.30}$ & 1 \\
\hline Q1206+1155 & 3.0230 & 18.90 & 19.50 & $19.20_{-0.30}^{+0.30}$ & 1 \\
\hline $\mathrm{J} 132729.75+484500$ & 3.0580 & 19.10 & 19.50 & $19.35_{-0.25}^{+0.25}$ & 1 \\
\hline SDSS1034+0358 & 3.0587 & 18.90 & 19.30 & $19.15_{-0.25}^{+0.15}$ & 1 \\
\hline Q2355+0108 & 3.0757 & 16.90 & 17.20 & $17.05_{-0.15}^{+0.15}$ & 1 \\
\hline SDSS0124+0044 & 3.0776 & 20.00 & 20.40 & $20.20_{-0.20}^{+0.20}$ & 1 \\
\hline Q2053-3546 & 3.0940 & 18.90 & 19.20 & $19.05_{-0.15}^{+0.15}$ & 1 \\
\hline Q0956+122 & 3.0963 & 17.08 & 17.28 & $17.18_{-0.10}^{+0.10}$ & 1 \\
\hline SDSS1621-0042 & 3.1050 & 19.60 & 20.00 & $19.80_{-0.20}^{+0.20}$ & 1 \\
\hline HE2218-6150 & 3.1106 & 16.80 & 17.20 & $17.00_{-0.20}^{+0.20}$ & 1 \\
\hline Q0336-01 & 3.1156 & 19.30 & 19.70 & $19.50_{-0.20}^{+0.20}$ & 1 \\
\hline SDSSJ2238-0921 & 3.1279 & 17.70 & 19.00 & $18.65_{-0.95}^{+0.35}$ & 2 \\
\hline SDSSJ1025+0452 & 3.1300 & 17.60 & 18.70 & $18.10_{-0.50}^{+0.50}$ & 2 \\
\hline $\mathrm{J} 1555+4800$ & 3.1310 & 19.45 & 19.75 & $19.60_{-0.15}^{+0.15}$ & 1 \\
\hline SDSS2315+1456 & 3.1350 & 19.80 & 20.10 & $19.95_{-0.15}^{+0.15}$ & 1 \\
\hline J0952+3329 & 3.1440 & 19.75 & 20.15 & $19.95_{-0.20}^{+0.20}$ & 1 \\
\hline $\mathrm{J} 1733+5400$ & 3.1510 & 17.90 & 19.10 & $18.70_{-0.80}^{+0.40}$ & 2 \\
\hline
\end{tabular}


Table 3

(Continued)

\begin{tabular}{|c|c|c|c|c|c|}
\hline Quasar & $z_{\text {peak }}{ }^{a}$ & $N_{\mathrm{HI}}^{\text {low }}$ & $N_{\mathrm{HI}}^{\text {high }}$ & $N_{\mathrm{H} \mathrm{I}}^{\text {adopt }}$ & $\mathrm{flg}_{\mathrm{H}}$ \\
\hline $\mathrm{J} 134939.78+124230.7$ & 3.1580 & 19.30 & 19.80 & $19.60_{-0.30}^{+0.30}$ & 1 \\
\hline Q2053-3546 & 3.1720 & 17.70 & 18.80 & $18.25_{-0.55}^{+0.55}$ & 2 \\
\hline PKS2000-330 & 3.1724 & 19.60 & 19.90 & $19.75_{-0.15}^{+0.15}$ & 1 \\
\hline SDSS2346-0016 & 3.1760 & 16.70 & 17.20 & $16.95_{-0.25}^{+0.25}$ & 1 \\
\hline PKS2000-330 & 3.1879 & 19.75 & 20.01 & $19.88_{-0.13}^{+0.13}$ & 1 \\
\hline J0952+3329 & 3.2112 & 19.70 & 20.10 & $19.90_{-0.20}^{+0.20}$ & 1 \\
\hline J092459.91+095103 & 3.2190 & 19.10 & 19.50 & $19.30_{-0.20}^{+0.20}$ & 1 \\
\hline SDSSJ2334-0908 & 3.2260 & 17.60 & 17.90 & $17.70_{-0.10}^{+0.30}$ & 1 \\
\hline $\mathrm{J} 1454+5114$ & 3.2310 & 19.90 & 20.20 & $20.05_{-0.15}^{+0.15}$ & 1 \\
\hline SDSS $1136+0050$ & 3.2483 & 17.50 & 18.75 & $18.10_{-0.60}^{+0.60}$ & 2 \\
\hline J0952+3329 & 3.2618 & 19.70 & 20.30 & $20.00_{-0.30}^{+0.30}$ & 1 \\
\hline SDSS $1040+5724$ & 3.2660 & 17.70 & 18.90 & $18.30_{-0.60}^{+0.60}$ & 2 \\
\hline PSS2323+2758 & 3.2670 & 18.60 & 19.80 & $19.40_{-0.80}^{+0.40}$ & 2 \\
\hline $\mathbf{J} 1337+0128$ & 3.2701 & 19.80 & 20.10 & $19.95_{-0.15}^{+0.15}$ & 1 \\
\hline SDSS0106+0048 & 3.2862 & 18.80 & 19.30 & $19.05_{-0.25}^{+0.25}$ & 1 \\
\hline SDSS0949+0355 & 3.3114 & 19.70 & 20.00 & $19.85_{-0.15}^{+0.15}$ & 1 \\
\hline SDSS2303-0939 & 3.3119 & 17.70 & 18.10 & $17.90_{-0.20}^{+0.20}$ & 1 \\
\hline SDSS0106+0048 & 3.3210 & 18.90 & 19.30 & $19.10_{-0.20}^{+0.20}$ & 1 \\
\hline SDSS0816+4823 & 3.3429 & 17.80 & 18.80 & $18.30_{-0.50}^{+0.50}$ & 2 \\
\hline SDSS0157-0106 & 3.3854 & 17.60 & 19.10 & $18.50_{-0.90}^{+0.60}$ & 2 \\
\hline $\mathrm{J} 1134+5742$ & 3.4100 & 17.78 & 18.16 & $17.97_{-0.19}^{+0.19}$ & 1 \\
\hline Q1330+0108 & 3.4210 & 19.00 & 19.40 & $19.20_{-0.20}^{+0.20}$ & 1 \\
\hline SDSS1429-0145 & 3.4266 & 17.60 & 18.40 & $18.00_{-0.40}^{+0.40}$ & 1 \\
\hline SDSS1402+0146 & 3.4558 & 18.90 & 19.40 & $19.20_{-0.30}^{+0.20}$ & 1 \\
\hline SDSS0810+4603 & 3.4720 & 19.60 & 20.20 & $19.90_{-0.30}^{+0.30}$ & 1 \\
\hline SDSS1110+0244 & 3.4759 & 17.70 & 18.50 & $18.10_{-0.40}^{+0.40}$ & 1 \\
\hline Q1108-0747 & 3.4810 & 19.80 & 20.20 & $20.00_{-0.20}^{+0.20}$ & 1 \\
\hline SDSS1249-0159 & 3.5302 & 17.70 & 18.40 & $18.10_{-0.40}^{+0.40}$ & 1 \\
\hline Q2000-330 & 3.5479 & 17.79 & 18.26 & $18.03_{-0.23}^{+0.23}$ & 1 \\
\hline PSS2323+2758 & 3.5649 & 18.80 & 19.50 & $19.25_{-0.45}^{+0.25}$ & 2 \\
\hline PSS1253-02 & 3.6031 & 19.15 & 19.55 & $19.35_{-0.20}^{+0.20}$ & 1 \\
\hline J073149.50+285448 & 3.6080 & 17.70 & 18.60 & $18.15_{-0.45}^{+0.45}$ & 2 \\
\hline PSS2241+1352 & 3.6539 & 20.00 & 20.40 & $20.20_{-0.20}^{+0.20}$ & 1 \\
\hline PSS1159+13 & 3.7230 & 19.75 & 20.05 & $19.90_{-0.15}^{+0.15}$ & 1 \\
\hline PX0034+16 & 3.7540 & 19.85 & 20.25 & $20.05_{-0.20}^{+0.20}$ & 1 \\
\hline $\mathrm{J} 132554.12+125546$ & 3.7670 & 19.40 & 19.80 & $19.60_{-0.20}^{+0.20}$ & 1 \\
\hline Q1108-0747 & 3.8112 & 17.70 & 18.70 & $18.20_{-0.50}^{+0.50}$ & 2 \\
\hline J034402.85-065300.6 & 3.8428 & 19.40 & 19.70 & $19.55_{-0.15}^{+0.15}$ & 1 \\
\hline SDSS0949+0355 & 3.9286 & 17.00 & 17.30 & $17.15_{-0.15}^{+0.15}$ & 1 \\
\hline J024447.78-081606.1 & 3.9529 & 16.75 & 17.15 & $16.95_{-0.20}^{+0.20}$ & 1 \\
\hline PSS0209+0517 & 3.9880 & 17.70 & 18.30 & $18.00_{-0.30}^{+0.30}$ & 1 \\
\hline PSS0133+0400 & 3.9950 & 19.80 & 20.30 & $20.10_{-0.30}^{+0.20}$ & 1 \\
\hline PSS1248+31 & 4.0751 & 19.80 & 20.10 & $19.95_{-0.15}^{+0.15}$ & 1 \\
\hline PSS1506+522 & 4.1140 & 17.60 & 18.90 & $18.50_{-0.90}^{+0.40}$ & 2 \\
\hline PSS0133+0400 & 4.1170 & 17.80 & 19.40 & $18.60_{-0.80}^{+0.80}$ & 2 \\
\hline SDSS0106+0048 & 4.1716 & 18.85 & 19.25 & $19.05_{-0.20}^{+0.20}$ & 1 \\
\hline BRJ0426-2202 & 4.1750 & 17.25 & 17.55 & $17.40_{-0.15}^{+0.15}$ & 1 \\
\hline PSS0134+3307 & 4.2789 & 17.55 & 17.85 & $17.70_{-0.15}^{+0.15}$ & 1 \\
\hline PSS1723+2243 & 4.3910 & 18.00 & 18.50 & $18.25_{-0.25}^{+0.25}$ & 1 \\
\hline
\end{tabular}

Notes. All column densities are $\log _{10}$. The flag in the final column indicates the quality of the measurement. A flg $\mathrm{HI}_{\mathrm{I}}=1$ corresponds to a more precisely measured value and one may assume a Gaussian PDF with the errors reported taken as $1 \sigma$ uncertainties. A flg $\mathrm{H}_{\mathrm{I}}=2$ corresponds to a less precisely measured value, and we recommend one adopt a uniform prior for $N_{\mathrm{HI}}$ within the error interval reported. See text for further details.

${ }^{a}$ Redshift estimates for the peak $\mathrm{H}_{\mathrm{I}}$ opacity from metals and Lyman series absorption. ions of the same element (e.g., $\mathrm{C}^{+3} / \mathrm{C}^{+}$) to eliminate offsets due to differing intrinsic chemical abundances (i.e., varying abundance ratios). In this analysis, we have taken the integrated column density across the entire LLS. While there is evidence for variations in these ratios within individual components, these tend to be small (e.g., Prochter et al. 2010, Figure 5). Therefore, the trends apparent in Figure 9 reflect the gross properties of the LLS sample.

All of the $\mathrm{C}^{+3} / \mathrm{C}^{+}, \mathrm{Si}^{+3} / \mathrm{Si}^{+}$, and $\mathrm{Al}^{++} / \mathrm{Al}^{+}$ratios exhibit a strong anti-correlation with $N_{\mathrm{H} \text { I }}$ indicating an increasing neutral fraction with increasing $\mathrm{H}_{\mathrm{I}}$ opacity. Taking limits as values, the Spearman rank test yields a probability of less than $10^{-3}$ for the null hypothesis, in each case. For all of these ions, the upper ionization state is dominant for $N_{\mathrm{H} \mathrm{I}} \lesssim 10^{18.5} \mathrm{~cm}^{-2}$ and vice-versa for higher $N_{\mathrm{H}_{\mathrm{I}}}$ values. We emphasize, however, that even at $N_{\mathrm{HI}} \approx 10^{20} \mathrm{~cm}^{-2}$ the observed ratios are frequently large, e.g., $\log \left(\mathrm{Si}^{+3} / \mathrm{Si}^{+}\right) \approx-0.5$ dex. This suggests that the gas is predominantly ionized even at these larger total H I opacities.

This inference is further supported by the $\mathrm{Si}^{+} / \mathrm{O}^{0}$. Ignoring differential depletion, which we expect to be modest in LLSs, the $\mathrm{Si}^{+} / \mathrm{O}^{0}$ ratio should trend toward the solar abundance ratio $\left(\epsilon_{\mathrm{Si}} / \epsilon_{\mathrm{O}}=-1.2 \mathrm{dex}\right)$ in a neutral gas given that $\mathrm{Si}$ and $\mathrm{O}$ are both produced in massive stars and are observed to trace each other in astrophysical systems (e.g., stellar atmospheres). We identify, however, a significant sample of systems with $N_{\mathrm{H} \text { I }} \approx 10^{18.5} \mathrm{~cm}^{-2}$ that have $\log \left(\mathrm{Si}^{+} / \mathrm{O}^{0}\right)>-1$ dex. Because the majority of ionization processes (e.g., photoionization, collisional ionization) predict $\mathrm{Si}^{+} / \mathrm{O}^{0}>\mathrm{Si} / \mathrm{O}$ (e.g., Prochaska \& Hennawi 2009), these measurements offer further evidence that LLSs are highly ionized.

\subsection{Metallicity}

A principal diagnostic of the LLSs is the gas metallicity, i.e., the enrichment of the gas in heavy elements. This quantity is generally characterized relative to the chemical abundances observed for the Sun. For the following, we adopt the solar abundance scale compiled by Asplund et al. (2009), taking meteoritic values when possible.

Because the LLSs are significantly ionized, the observed ionic abundances reflect only a fraction of the total abundances of $\mathrm{Si}, \mathrm{O}, \mathrm{H}$, etc. Therefore, a full treatment requires ionization modeling. We may, however, offer insight into the problem by examining several ions relative to $\mathrm{H}^{0}$. To minimize ionization corrections, one restricts the analysis to ionization states dominant in a highly optically thick (i.e., neutral) medium.

The results for four low-ions are presented in Figure 10, normalized to the solar abundance. We have introduced here a new quantity and notation: $\left\{\mathrm{X}^{i} / \mathrm{Y}^{j}\right\}$ $\equiv \log \left(N\left(\mathrm{X}^{i}\right) / N\left(\mathrm{Y}^{j}\right)\right)-\epsilon_{\mathrm{X}}+\epsilon_{\mathrm{Y}}$, where $\epsilon_{\mathrm{X}}$ is the solar abundance on the logarithmic scale for element $\mathrm{X}$. This quantity explicitly ignores ionization corrections and should not be considered a proper estimate of the chemical abundance ratio, traditionally expressed as $[\mathrm{X} / \mathrm{Y}]$. In the cases where ionization corrections are negligible, however, $\left\{\mathrm{X}^{i} / \mathrm{H}^{0}\right\}=[\mathrm{X} / \mathrm{H}]$ and this quantity represents the metallicity.

A cursory inspection of the plots suggests a significant decline in metal content with increasing $N_{\mathrm{H} \text { I }}$. This apparent anti-correlation, however, is driven by at least two factors. First, larger $N_{\mathrm{H}}$ implies larger metal column densities such that the transitions saturate yielding a preponderance of lower 


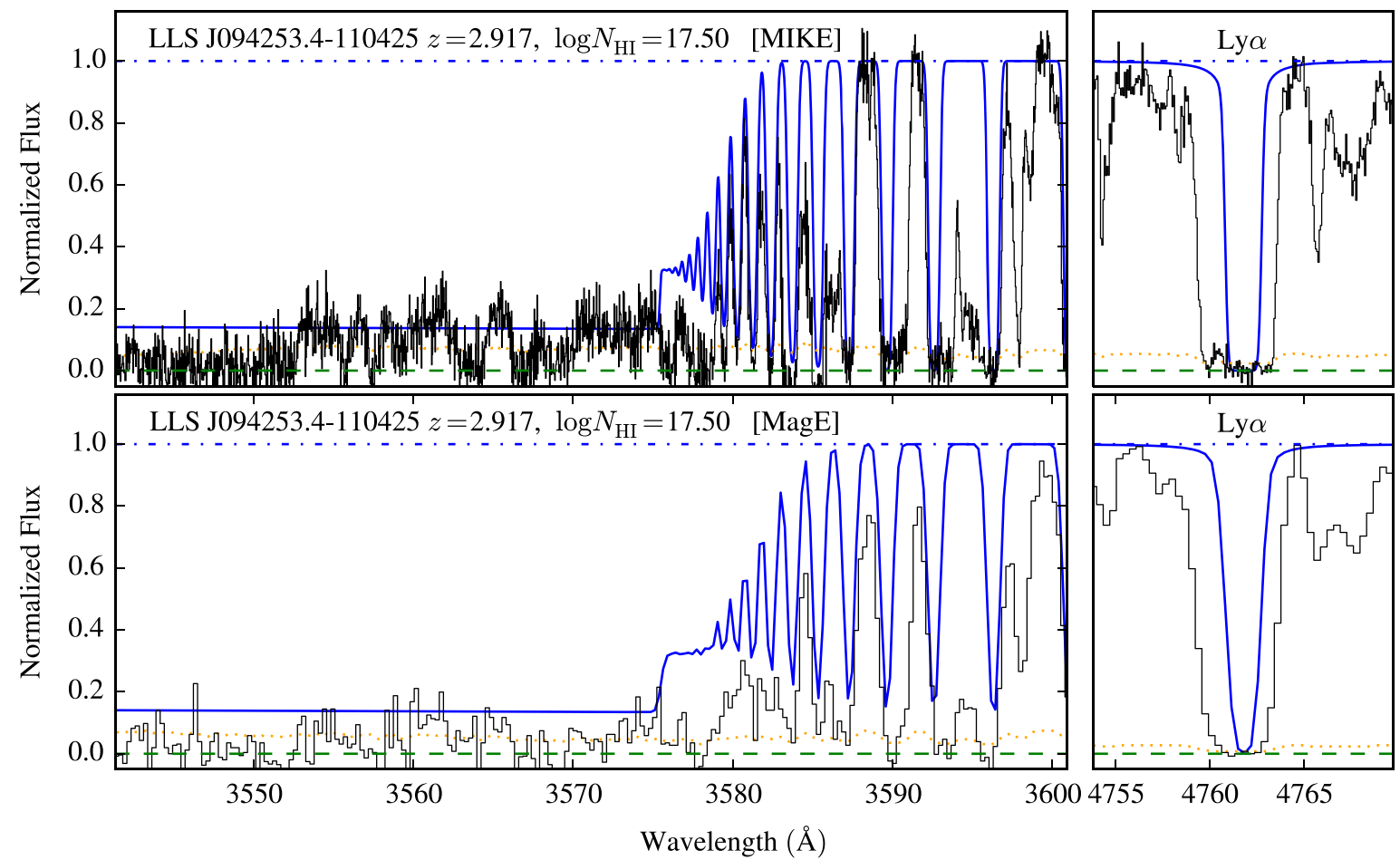

Figure 2. Plots of the Lyman limit (left) and the Ly $\alpha$ (right) profile for the LLS at $z=2.917$ toward J0924-1104. We observed this system with both the MIKE (top) and MagE (bottom) spectrometers at LCO. The spectra show a break in the flux at $\lambda \approx 3585 \AA$ but residual flux down to $\lambda \approx 3500 \AA$ where the Lyman limit from a lower redshift absorber occurs. By modeling the flux decrement of the $z=2.917 \mathrm{LLS}$, we establish a precise estimate of its total $N_{\mathrm{H} \text { I }}$ value. It is also evident that the H I Ly $\alpha$ profile alone offers very little constraint on $N_{\mathrm{HI}}$.
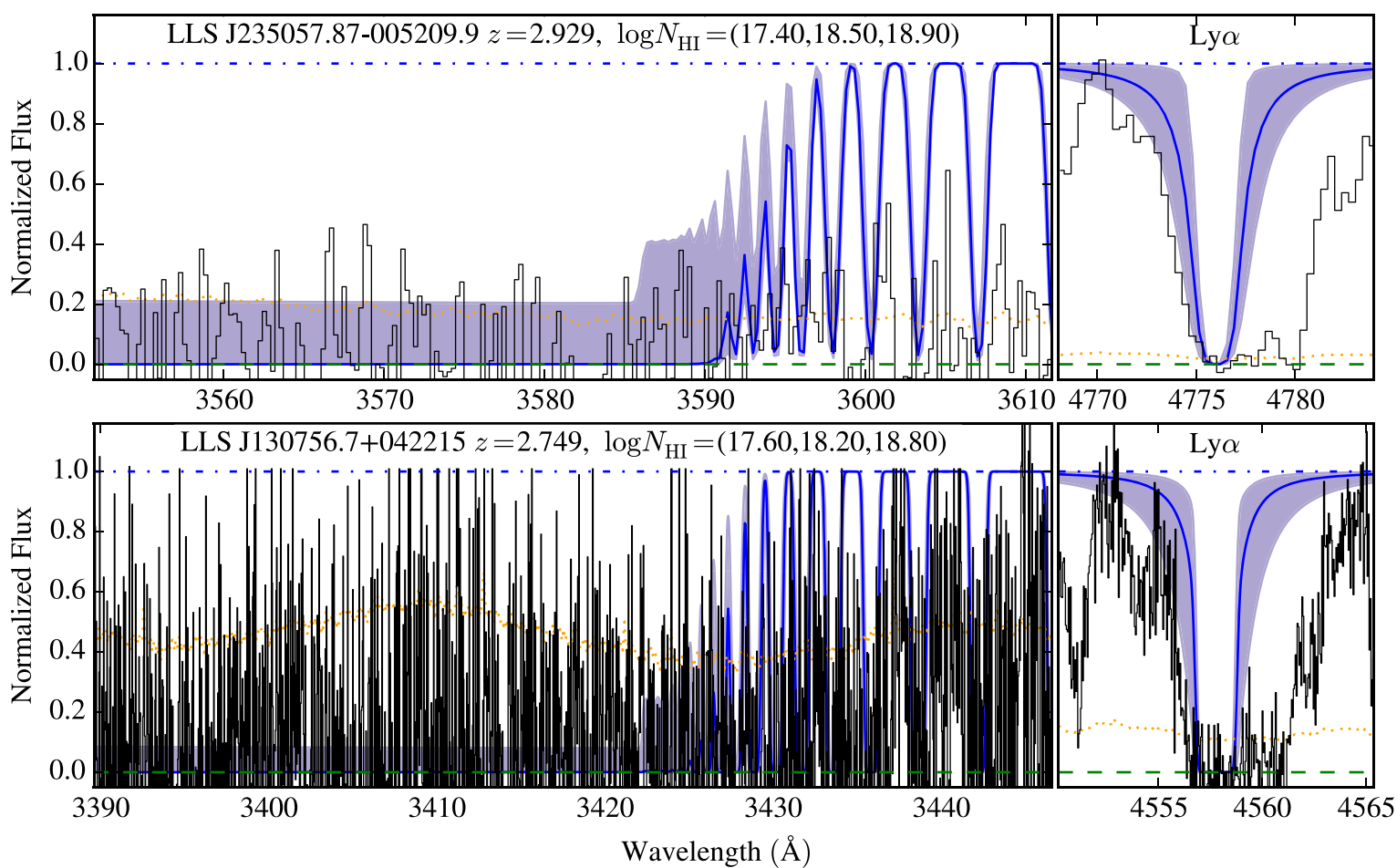

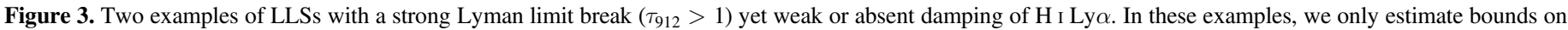
the $N_{\mathrm{H} \text { I }}$ values which can span an order of magnitude uncertainty.

limits. By the same token, at low $N_{\mathrm{H} \text { I }}$ values the transitions are often undetected yielding upper limits to the ionic ratios. Second, we have argued from Figure 9 that the gas is increasingly ionized with decreasing $N_{\mathrm{H}}$. For $\mathrm{Si}^{+}, \mathrm{C}^{+}$, and
$\mathrm{Al}^{+}$, the ionization corrections for $\left\{\mathrm{X}^{i} / \mathrm{H}^{0}\right\}$ are likely negative (e.g., Prochaska 1999; Fumagalli et al. 2011a), and would lower the metallicity one infers from such ratios. We believe these factors dominate the trends apparent in Figure 10. 

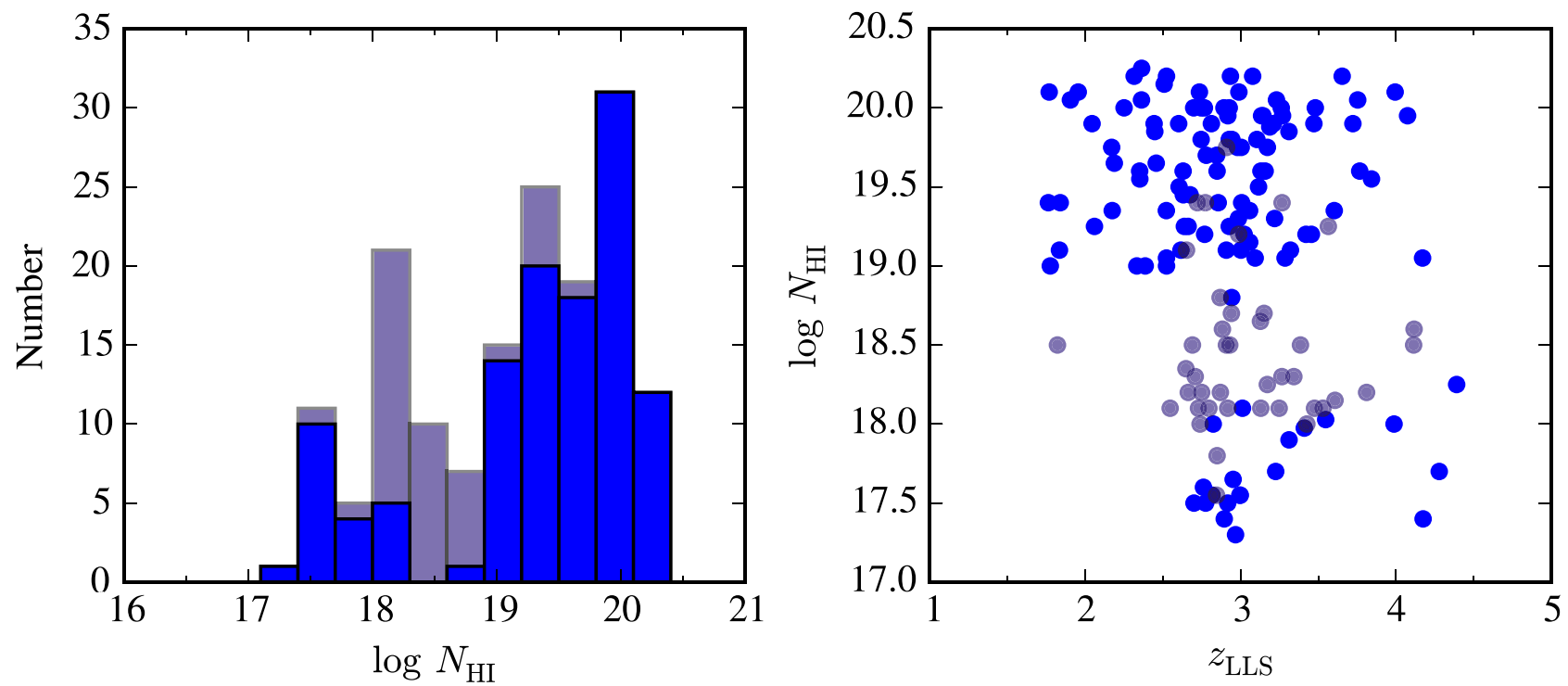

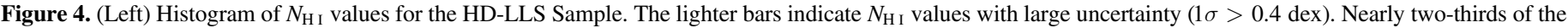

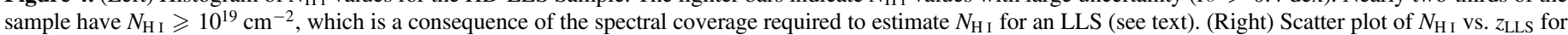

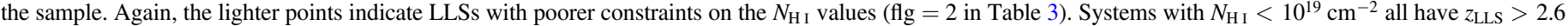
as coverage of the Lyman limit is required for the $\mathrm{H}$ I analysis.
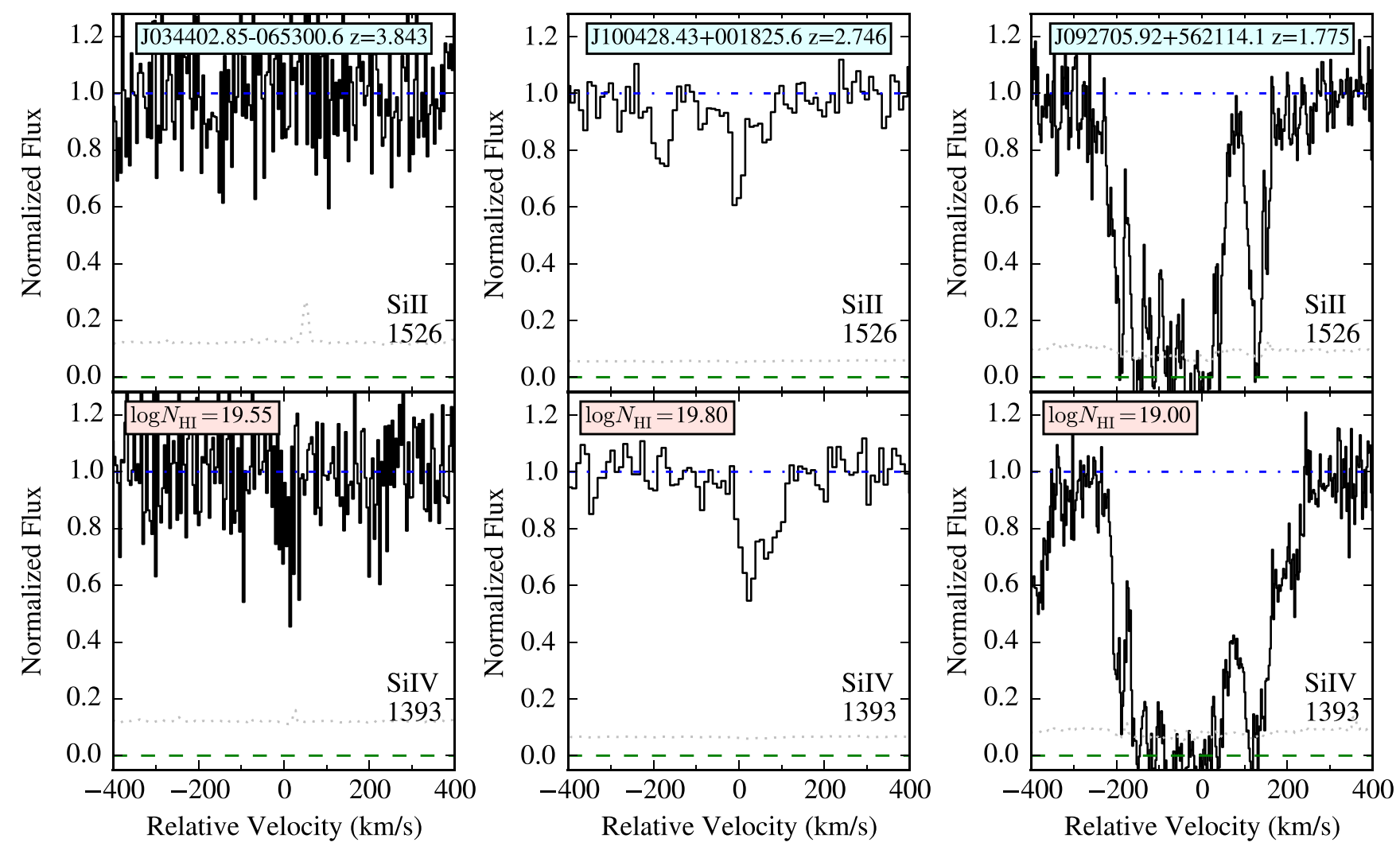

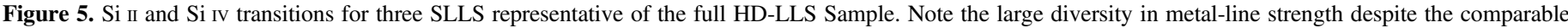

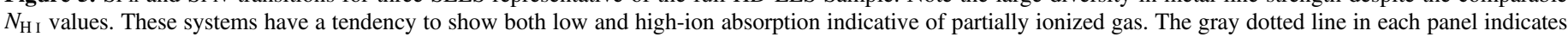
the estimated $1 \sigma$ error array.

In fact, it is even possible that the true distribution exhibits the opposite trend. Figure 11 shows $[\mathrm{O} / \mathrm{H}]$ against $N_{\mathrm{H}}$ for the LLSs where we have assumed no ionization corrections, i.e., $[\mathrm{O} / \mathrm{H}]=\left\{\mathrm{O}^{0} / \mathrm{H}^{0}\right\}$. This approximation is justified by the fact that $\mathrm{O}^{0}$ and $\mathrm{H}^{0}$ have very similar ionization potentials and their neutral states are coupled by charge-exchange reactions. This assumption may break down at low $N_{\mathrm{H}}$ values in the presence of a hard radiation field (Sofia \& Jenkins 1998; Prochter et al. 2010), but the corrections are still likely to be modest (several tenths dex). Unfortunately, the measurements are dominated by limits: upper limits at $N_{\mathrm{H}}<10^{18.5} \mathrm{~cm}^{-2}$ and lower limits at $N_{\mathrm{H}}>10^{19} \mathrm{~cm}^{-2}$. Nevertheless, the data 

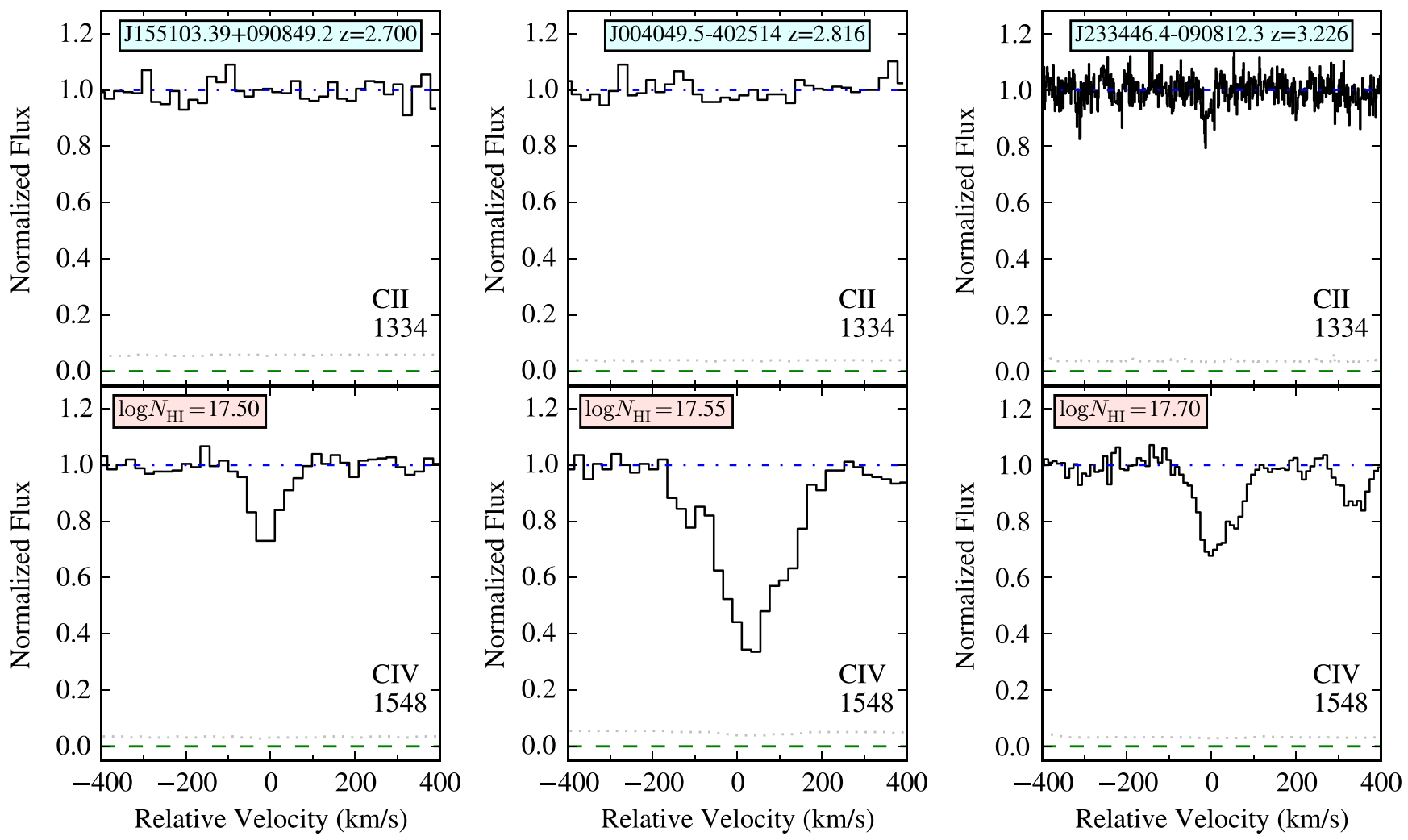

Figure 6. $\mathrm{C}$ II and $\mathrm{C}$ IV transitions for LLSs with low $N_{\mathrm{H}}$ values. Unlike the SLLSs from Figure 5, these LLSs have metal absorption that is dominated by high ions. There only a few cases of LLSs with $N_{\mathrm{H} \text { I }}<10^{18} \mathrm{~cm}^{-2}$ and a positive low-ion detection.

Table 4

Ionic Column Summary for Si and C

\begin{tabular}{|c|c|c|c|c|c|c|c|c|c|c|}
\hline Quasar & $z_{\mathrm{abs}}$ & $N_{\mathrm{H} \mathrm{I}}$ & $N\left(\mathrm{C}^{+}\right)$ & $\sigma\left(N\left(\mathrm{C}^{+}\right)\right)$ & $N\left(\mathrm{C}^{3+}\right)$ & $\sigma\left(N\left(\mathrm{C}^{3+}\right)\right)$ & $N\left(\mathrm{Si}^{+}\right)$ & $\sigma\left(N\left(\mathrm{Si}^{+}\right)\right)$ & $N\left(\mathrm{Si}^{3+}\right)$ & $\sigma\left(N\left(\mathrm{Si}^{3+}\right)\right)$ \\
\hline $\mathrm{J} 1608+0715$ & 1.7626 & $19.40_{-0.30}^{+0.30}$ & $\cdots$ & $\ldots$ & $\cdots$ & $\cdots$ & 15.80 & -9.99 & $\cdots$ & $\cdots$ \\
\hline $\mathrm{J} 0953+5230$ & 1.7678 & $20.10_{-0.10}^{+0.10}$ & 15.44 & +9.99 & 15.21 & +9.99 & 15.67 & 0.01 & 14.57 & +9.99 \\
\hline $\mathrm{J} 0927+5621$ & 1.7749 & $19.00_{-0.10}^{+0.10}$ & 15.40 & +9.99 & 15.40 & +9.99 & 15.58 & 0.02 & 14.84 & +9.99 \\
\hline $\mathrm{J} 1509+1113$ & 1.8210 & $18.50_{-0.50}^{+0.50}$ & $\ldots$ & $\ldots$ & 14.83 & +9.99 & 14.21 & 0.04 & 14.17 & +9.99 \\
\hline J101939.15+524627 & 1.8339 & $19.10_{-0.30}^{+0.30}$ & $\cdots$ & $\cdots$ & 14.93 & +9.99 & 15.32 & 0.03 & 14.14 & +9.99 \\
\hline Q1100-264 & 1.8389 & $19.40_{-0.15}^{+0.15}$ & $\cdots$ & $\cdots$ & 14.24 & 0.00 & 13.96 & 0.01 & 13.83 & 0.00 \\
\hline J1159-0032 & 1.9044 & $20.05_{-0.15}^{+0.15}$ & 15.38 & +9.99 & 15.22 & +9.99 & 15.14 & 0.10 & 14.54 & +9.99 \\
\hline Q0201+36 & 1.9548 & $20.10_{-0.20}^{+0.20}$ & $\cdots$ & $\cdots$ & $\cdots$ & $\cdots$ & 15.11 & 0.09 & $\cdots$ & $\cdots$ \\
\hline $\mathrm{J} 0828+0858$ & 2.0438 & $19.90_{-0.10}^{+0.10}$ & 15.14 & +9.99 & 14.89 & +9.99 & 15.25 & 0.10 & 14.44 & +9.99 \\
\hline $\mathrm{J} 2123-0050$ & 2.0593 & $19.25_{-0.15}^{+0.15}$ & 15.11 & +9.99 & 14.60 & +9.99 & 14.60 & 0.04 & 13.96 & 0.00 \\
\hline Q1456-1938 & 2.1701 & $19.75_{-0.20}^{+0.20}$ & $\cdots$ & $\ldots$ & $\cdots$ & $\ldots$ & 14.84 & -9.99 & $\ldots$ & $\ldots$ \\
\hline J034024.57-051909 & 2.1736 & $19.35_{-0.20}^{+0.20}$ & 14.40 & +9.99 & 13.86 & 0.02 & 13.84 & 0.02 & 13.39 & 0.02 \\
\hline Q0001-2340 & 2.1871 & $19.65_{-0.15}^{+0.15}$ & 14.45 & +9.99 & 14.26 & 0.01 & 13.75 & 0.03 & 13.74 & 0.01 \\
\hline SDSS1307+0422 & 2.2499 & $20.00_{-0.15}^{+0.15}$ & $\ldots$ & $\ldots$ & 14.22 & 0.03 & 14.25 & +9.99 & $\ldots$ & $\cdots$ \\
\hline $\mathrm{J} 1712+5755$ & 2.3148 & $20.20_{-0.15}^{+0.15}$ & $\cdots$ & $\cdots$ & 13.36 & 0.04 & 14.08 & 0.01 & $\cdots$ & $\cdots$ \\
\hline
\end{tabular}

Note. All column densities are $\log _{10}$. When the reported $\sigma=+9.99$, the measured value should be taken as a lower limit. Similarly, $\sigma=-9.99$ indicates that the reported value refers to an upper limit at $95 \%$ c.l.

(This table is available in its entirety in machine-readable form.)

require that $[\mathrm{O} / \mathrm{H}]>-1.7$ for the SLLSs and indicate $[\mathrm{O} / \mathrm{H}]$ $<-1.3$ dex for LLSs with $N_{\mathrm{HI}} \approx 10^{18} \mathrm{~cm}^{-2}$. We tentatively infer that the median metallicity is approximately flat with $N_{\mathrm{H} \text { I }}$ and possibly increasing; more strictly, we rule out a steeply declining $\mathrm{O} / \mathrm{H}$ metallicity with increasing $N_{\mathrm{HI}}$. A similar conclusion may be drawn from the $\left\{\mathrm{Si}^{+} / \mathrm{H}^{0}\right\}$ measurements which scatter less from line-saturation. The LLSs with $N_{\mathrm{H} \text { I }} \approx 10^{18} \mathrm{~cm}^{-2}$ show very few positive detections and have a median $\left\{\mathrm{Si}^{+} / \mathrm{H}^{0}\right\}<-1 \mathrm{dex}$. In contrast, the LLSs at $N_{\mathrm{H}} \approx 10^{19.5} \mathrm{~cm}^{-2}$ frequently exhibit $\left\{\mathrm{Si}^{+} / \mathrm{H}^{0}\right\}>-1$ dex.

Another result apparent from Figure 10 is the large dispersion in measurements at every $N_{\mathrm{H} \text { I }}$ value. This is most notable for $\mathrm{Si}^{+}$which has multiple transitions that permit measurements of the column density over a larger dynamic

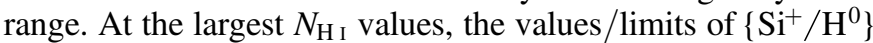
span nearly four orders of magnitude! And although the 
Table 5

Ionic Column Summary For Al, Fe, and O

\begin{tabular}{|c|c|c|c|c|c|c|c|c|c|c|}
\hline Quasar & $z_{\mathrm{abs}}$ & $N_{\mathrm{H} \mathrm{I}}$ & $N\left(\mathrm{O}^{0}\right)$ & $\sigma\left(N\left(\mathrm{O}^{0}\right)\right)$ & $N\left(\mathrm{Al}^{+}\right)$ & $\sigma\left(N\left(\mathrm{Al}^{+}\right)\right)$ & $N\left(\mathrm{Al}^{++}\right)$ & $\sigma\left(N\left(\mathrm{Al}^{++}\right)\right)$ & $N\left(\mathrm{Fe}^{+}\right)$ & $\sigma\left(N\left(\mathrm{Fe}^{+}\right)\right)$ \\
\hline $\mathrm{J} 1608+0715$ & 1.7626 & $19.40_{-0.30}^{+0.30}$ & $\cdots$ & $\ldots$ & $\ldots$ & $\ldots$ & 13.53 & 0.00 & $\cdots$ & $\ldots$ \\
\hline J0953+5230 & 1.7678 & $20.10_{-0.10}^{+0.10}$ & 15.68 & +9.99 & 13.96 & +9.99 & 13.86 & 0.01 & 14.99 & 0.10 \\
\hline $\mathrm{J} 1509+1113$ & 1.8210 & $18.50_{-0.50}^{+0.50}$ & $\ldots$ & $\ldots$ & 13.12 & +9.99 & 13.04 & 0.05 & 13.76 & 0.11 \\
\hline J101939.15+524627 & 1.8339 & $19.10_{-0.30}^{+0.30}$ & $\ldots$ & $\ldots$ & 13.34 & +9.99 & 13.62 & 0.02 & 14.19 & 0.02 \\
\hline Q1100-264 & 1.8389 & $19.40_{-0.15}^{+0.15}$ & $\cdots$ & $\cdots$ & 12.79 & 0.01 & 12.31 & 0.10 & 13.42 & 0.01 \\
\hline J0828+0858 & 2.0438 & $19.90_{-0.10}^{+0.10}$ & 15.49 & +9.99 & $\ldots$ & $\cdots$ & 13.59 & 0.02 & 14.89 & 0.04 \\
\hline J2123-0050 & 2.0593 & $19.25_{-0.15}^{+0.15}$ & $\cdots$ & $\cdots$ & 13.44 & +9.99 & 13.15 & 0.01 & 14.39 & 0.00 \\
\hline Q1456-1938 & 2.1701 & $19.75_{-0.20}^{+0.20}$ & $\cdots$ & $\cdots$ & 13.38 & +9.99 & 12.99 & 0.04 & 14.26 & 0.01 \\
\hline J034024.57-051909 & 2.1736 & $19.35_{-0.20}^{+0.20}$ & 14.56 & +9.99 & 12.65 & 0.03 & 12.49 & 0.14 & $\ldots$ & $\cdots$ \\
\hline Q0001-2340 & 2.1871 & $19.65_{-0.15}^{+0.15}$ & 14.16 & 0.04 & 13.00 & +9.99 & 12.40 & -9.99 & 13.11 & 0.03 \\
\hline SDSS1307+0422 & 2.2499 & $20.00_{-0.15}^{+0.15}$ & $\ldots$ & $\ldots$ & 13.04 & +9.99 & 12.80 & 0.07 & 14.18 & 0.04 \\
\hline
\end{tabular}

Note. All column densities are $\log _{10}$. When the reported $\sigma=+9.99$, the measured value should be taken as a lower limit. Similarly, $\sigma=-9.99$ indicates that the reported value refers to an upper limit at $95 \%$ c.l.

(This table is available in its entirety in machine-readable form.)
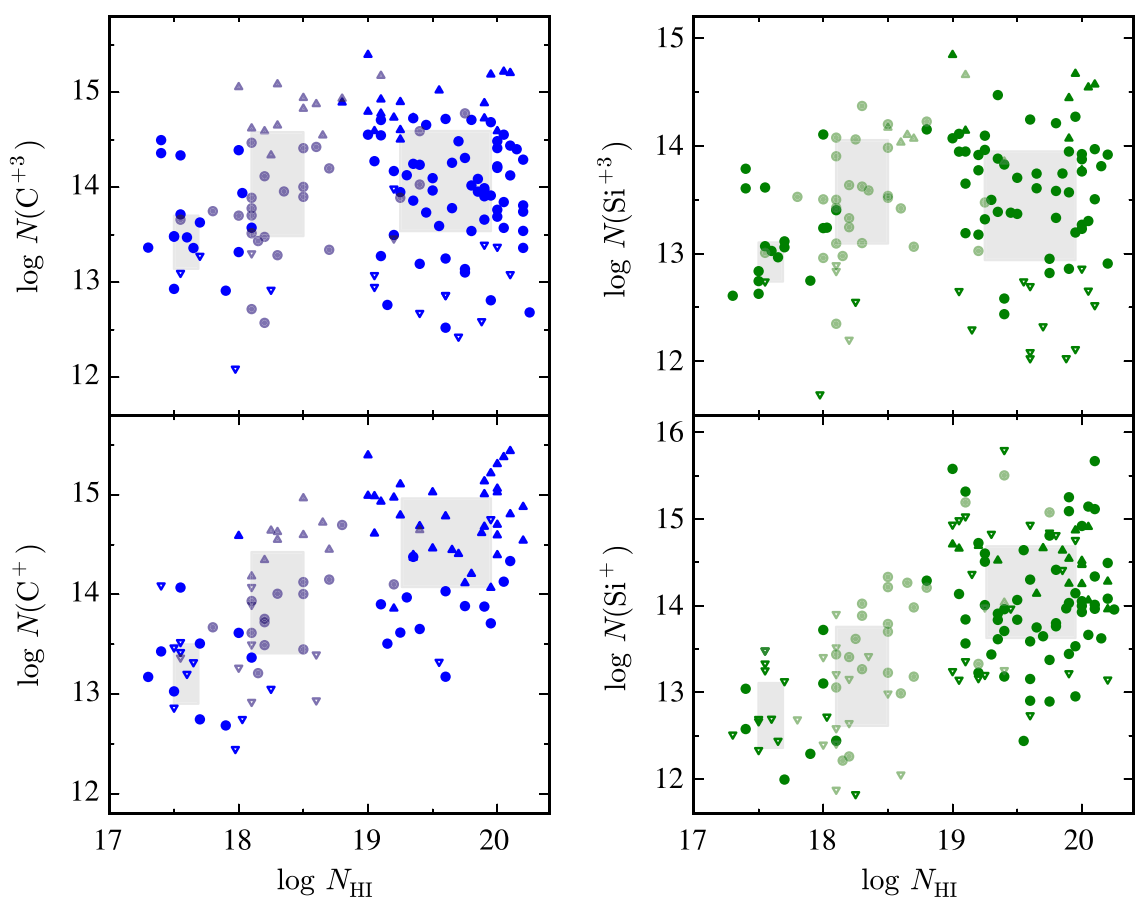

Figure 7. Scatter plot of Si and C ionic column densities for the HD-LLS Sample. Circles indicate measured values; their uncertainties are generally less than 0.1 dex. Triangles indicate limits to the values with the open symbols indicating upper limits. Lighter points mark LLSs with a poorly constrained $N_{\mathrm{H} \text { I }}$ value. Gray boxes encompass $50 \%$ of the measurements in three logarithmic $N_{\mathrm{HI}}$ intervals: $[17.3,18.0),[18.0,19.0),[19.0,20.3)$. At all column densities, there is a large dispersion in the measurements. Nevertheless, the low ions $\left(\mathrm{C}^{+}, \mathrm{Si}^{+}\right)$exhibit a strong, positive correlation with $N_{\mathrm{H} \text { I }}$ value. A Spearman rank test rules out the null hypothesis at $>99.99 \%$ c.l.

measurements for LLSs with $N_{\mathrm{HI}} \approx 10^{17.5}-10^{19} \mathrm{~cm}^{-2}$ include many upper limits, one identifies values and upper limits with $\left\{\mathrm{Si}^{+} / \mathrm{H}^{0}\right\}>-0.5$ dex together with upper limits having $\left\{\mathrm{Si}^{+} / \mathrm{H}^{0}\right\}<-2$. Clearly, any underlying trend of enrichment with $N_{\mathrm{H} \text { I }}$ will be diluted by the large intrinsic scatter within the LLSs. One may even argue that if such a dispersion is indicative of multiple astrophysical systems, then defining a mean of the LLS population has limited scientific value.
Despite the large dispersion, we emphasize that very few of the LLS in the HD-LLS Sample are "metal-free," i.e., exhibiting no metal-line absorption and therefore consistent with primordial abundances. Of the $n_{\text {LLS }}$ LLSs, only 25 have no low-ion detections outside the $\operatorname{Ly} \alpha$ forest and 18 of these exhibit a positive detection in a higher-ion. For the other 7 , one has been previously been identified as consistent with primordial (Fumagalli et al. 2011a). The remainder have a 

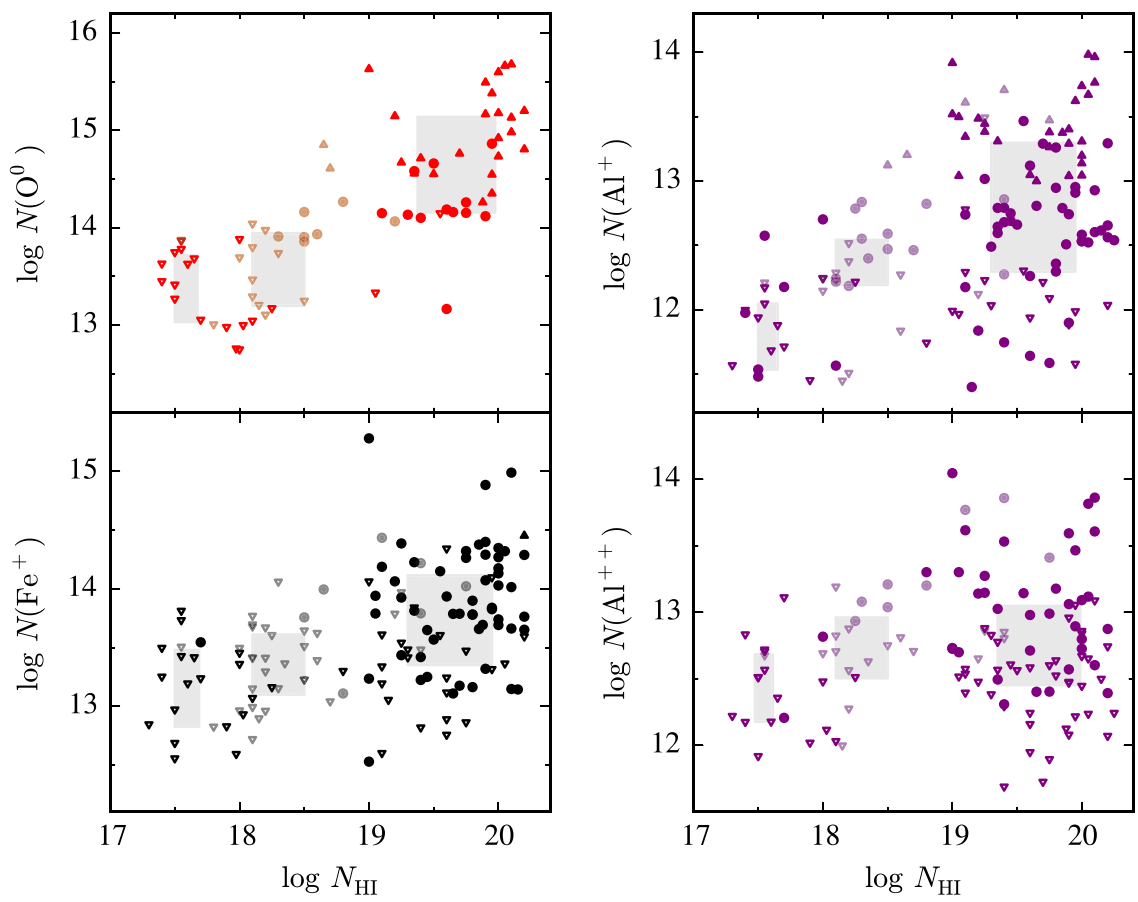

Figure 8. Same as Figure 7 but for four additional ions.

diversity of $\mathrm{S} / \mathrm{N}$ and spectral coverage and therefore are generally less sensitive to measuring a low metallicity. Several will be examined in greater detail in a future manuscript. Nevertheless, we may conclude that the incidence of very low metallicity gas $(<1 / 1000$ solar $)$ is rare in the LLS population $(<5 \%)$. Furthermore, none of the 82 LLSs with $N_{\mathrm{H} \mathrm{I}}>10^{19.2} \mathrm{~cm}^{-2}$ are metal-free. ${ }^{11}$ By $z \sim 3$, gas that is dense enough to exhibit a very high Lyman limit opacity has previously been polluted by heavy elements.

At the opposite end of the enrichment distribution, we identify 13 systems with a positive $\left\{\mathrm{Si}^{+} / \mathrm{H}^{0}\right\}$ measurement that exceeds 0 dex. This includes four extreme examples with $\left\{\mathrm{Si}^{+} / \mathrm{H}^{0}\right\}$ $>+0.5$ dex. Because these four LLSs also have $N_{\mathrm{H}} \geqslant 10^{19} \mathrm{~cm}^{-2}$, we expect that corrections for ionization are modest (see Prochaska et al. 2006) and that these are truly supersolar abundances. The others, however, have uncertainties consistent with the gas being sub-solar even before accounting for ionization. We conclude, subject to additional future analysis, that super-solar enrichment is also rare in the LLSs.

In Figure 12, we examine $\left\{\mathrm{Si}^{+} / \mathrm{H}^{0}\right\}$ and $\left\{\mathrm{C}^{+} / \mathrm{H}^{0}\right\}$ values as a function of redshift, splitting the LLS sample at $N_{\mathrm{H} \text { I }}=10^{19} \mathrm{~cm}^{-2}$. The values for the lower $N_{\mathrm{H} \text { I }}$ systems suggest a declining trend with increasing redshift, e.g., in contrast to the lower redshift systems, none of the $z>3.5$ LLSs have a positive detection of $\left\{\mathrm{C}^{+} / \mathrm{H}^{0}\right\}>-0.5$ dex. Even if we restrict analysis to positive detections, however, an anticorrelation is not statistically significant.

Turning to the SLLS population, the $\left\{\mathrm{X}^{i} / \mathrm{H}^{0}\right\}$ distributions show obvious trends with redshift (limits not withstanding). Treating all of the positive detections at their plotted values, a Spearman's rank correlation test rules out the null hypothesis at $>99 \%$ c.l. We interpret this anti-correlation as lower average enrichment within the SLLS at higher redshift. This conclusion

\footnotetext{
11 There is the possibility of a slight bias against our identifying metal-free SLLS but we have been as careful as possible to select systems based solely on the Ly $\alpha$ profile.
}

relies on the assumption that ionization corrections will not evolve significantly with redshift, which will be investigated in a future work. A similar decline in metallicity has been established in the DLA population (e.g., Prochaska et al. 2003a; Rafelski et al. 2012) and has been interpeted as resulting from the ongoing enrichment of galactic ISM with cosmic time. Future work will perform a quantitative comparison between the two populations and explore the implications for the evolving enrichment of optically thick gas at $z>2$. In passing, we emphasize the absence of low $\left\{\mathrm{X}^{i} / \mathrm{H}^{0}\right\}$ values at $z \approx 2$ which implies a reduced incidence of nearpristine gas with high $\mathrm{H}$ i columns at that epoch.

\subsection{Nucleosynthetic Patterns}

It is the conventional wisdom that LLSs primarily trace gas outside of the ISM of galaxies, e.g., within their dark matter halos (aka CGM) or at yet greater distances (Fumagalli et al. 2011b; Prochaska et al. 2013). Despite their separation from galaxies, we have demonstrated that the LLSs are generally enriched in heavy elements and provided evidence that their metallicity frequently reaches $\sim 1 / 10$ solar abundance. Therefore, a non-negligible fraction of this optically thick medium has been processed through the furnaces of a stellar interior and presumably was transported from a galaxy via one more physical processes. One plausible transport process is an explosive event, e.g., a supernovae that expelled the gas shortly after enriching it. In this case, the gas may exhibit a distinct nucleosynthetic pattern from those observed for galactic ISM, i.e., if the supernovae ejecta did not mix prior to escaping the system. Additionally, the LLSs may couple the metal production within galaxies to the enrichment of the diffuse IGM (e.g., Aguirre et al. 2001; Schaye et al. 2003; Steidel et al. 2010). This motivates comparison of the abundances for these two diffuse and ionized phases.

We may explore several ionic ratios that trace different nucleosynthesis channels. As with metallicity, one must 
Table 6

Ionic Column Densities

\begin{tabular}{|c|c|c|c|c|c|c|c|c|c|c|c|c|c|}
\hline Quasar & $\begin{array}{l}\text { R.A. } \\
\text { (J2000) }\end{array}$ & $\begin{array}{l}\text { decl. } \\
\text { (J2000) }\end{array}$ & $z_{\mathrm{abs}}$ & $N_{\mathrm{H} \mathrm{I}}$ & $\begin{array}{l}\lambda \\
(\AA)\end{array}$ & $\begin{array}{c}v_{\lim } \\
\left(\mathrm{km} \mathrm{s}^{-1}\right)\end{array}$ & flg & $N_{\lambda}$ & $\sigma(N)$ & Ion & flg & $N_{\text {ion }}$ & $\sigma(N)$ \\
\hline \multirow[t]{30}{*}{ Q0001-2340 } & $00: 03: 45$ & $-23: 23: 46.5$ & 2.18710 & 19.65 & 1334.5323 & $-423,64$ & 2 & 14.45 & 99.99 & 6,2 & 2 & 14.45 & 99.99 \\
\hline & & & & & 1335.7077 & $-205,64$ & 4 & 13.10 & 99.99 & & & & \\
\hline & & & & & 1548.1950 & $-423,64$ & 0 & 14.26 & 0.01 & 6,4 & 1 & 14.26 & 0.05 \\
\hline & & & & & 1550.7700 & $-394,64$ & 0 & 14.25 & 0.02 & & & & \\
\hline & & & & & 1302.1685 & $-213,64$ & 0 & 14.16 & 0.04 & 8,1 & 1 & 14.16 & 0.05 \\
\hline & & & & & 2852.9642 & $-213,64$ & 4 & 11.72 & 99.99 & 12,1 & 3 & 11.72 & 99.99 \\
\hline & & & & & 2796.3520 & $-413,64$ & 2 & 13.43 & 99.99 & 12,2 & 1 & 13.64 & 0.05 \\
\hline & & & & & 2803.5310 & $-404,64$ & 0 & 13.65 & 0.02 & & & & \\
\hline & & & & & 1670.7874 & $-405,64$ & 2 & 13.00 & 99.99 & 13,2 & 2 & 13.00 & 99.99 \\
\hline & & & & & 1854.7164 & $-213,64$ & 4 & 12.40 & 99.99 & 13,3 & 3 & 12.40 & 99.99 \\
\hline & & & & & 1862.7895 & $-213,64$ & 4 & 12.69 & 99.99 & & & & \\
\hline & & & & & 1260.4221 & $-399,64$ & 2 & 13.81 & 99.99 & 14,2 & 1 & 13.75 & 0.05 \\
\hline & & & & & 1304.3702 & $-423,64$ & 0 & 13.55 & 0.09 & & & & \\
\hline & & & & & 1526.7066 & $-399,64$ & 0 & 13.83 & 0.03 & & & & \\
\hline & & & & & 1808.0130 & $-213,64$ & 4 & 14.86 & 99.99 & & & & \\
\hline & & & & & 1393.7550 & $-411,64$ & 0 & 13.78 & 0.01 & 14,4 & 1 & 13.74 & 0.05 \\
\hline & & & & & 1402.7700 & $-421,64$ & 0 & 13.64 & 0.02 & & & & \\
\hline & & & & & 1250.5840 & $-79,64$ & 0 & 14.19 & 0.13 & 16,2 & 1 & 14.19 & 0.13 \\
\hline & & & & & 1608.4511 & $-213,64$ & 4 & 13.49 & 99.99 & 26,2 & 1 & 13.11 & 0.05 \\
\hline & & & & & 2344.2140 & $-213,64$ & 0 & 13.22 & 0.07 & & & & \\
\hline & & & & & 2374.4612 & $-213,64$ & 4 & 13.46 & 99.99 & & & & \\
\hline & & & & & 2382.7650 & $-213,64$ & 0 & 13.01 & 0.04 & & & & \\
\hline & & & & & 2586.6500 & $-213,64$ & 4 & 13.15 & 99.99 & & & & \\
\hline & & & & & 2600.1729 & $-328,64$ & 0 & 13.25 & 0.04 & & & & \\
\hline & & & & & 1317.2170 & $-213,64$ & 4 & 13.41 & 99.99 & 28,2 & 3 & 13.40 & 99.99 \\
\hline & & & & & 1370.1310 & $-213,64$ & 4 & 13.40 & 99.99 & & & & \\
\hline & & & & & 1454.8420 & $-213,64$ & 4 & 13.56 & 99.99 & & & & \\
\hline & & & & & 1741.5531 & $-213,64$ & 4 & 13.60 & 99.99 & & & & \\
\hline & & & & & 1751.9157 & $-213,64$ & 4 & 13.83 & 99.99 & & & & \\
\hline & & & & & 2026.1360 & $-213,64$ & 4 & 12.38 & 99.99 & 30,2 & 3 & 12.38 & 99.99 \\
\hline \multirow[t]{11}{*}{ PX0034+16 } & $00: 34: 54.8$ & $+16: 39: 20$ & 3.75397 & 20.05 & 1548.1950 & $-242,65$ & 0 & 13.85 & 0.02 & 6,4 & 1 & 13.85 & 0.05 \\
\hline & & & & & 1550.7700 & $-118,189$ & 2 & 13.68 & 99.99 & & & & \\
\hline & & & & & 1670.7874 & $-187,189$ & 0 & 12.52 & 0.04 & 13,2 & 1 & 12.52 & 0.05 \\
\hline & & & & & 1854.7164 & $-115,120$ & 4 & 12.24 & 99.99 & 13,3 & 3 & 12.24 & 99.99 \\
\hline & & & & & 1862.7895 & $-89,129$ & 4 & 12.57 & 99.99 & & & & \\
\hline & & & & & 1526.7066 & $-54,122$ & 2 & 14.06 & 99.99 & 14,2 & 2 & 14.06 & 99.99 \\
\hline & & & & & 1808.0130 & $-133,138$ & 4 & 14.73 & 99.99 & & & & \\
\hline & & & & & 1393.7550 & $-160,133$ & 0 & 13.30 & 0.02 & 14,4 & 1 & 13.30 & 0.05 \\
\hline & & & & & 1741.5531 & $-187,189$ & 4 & 14.29 & 99.99 & 28,2 & 3 & 13.72 & 99.99 \\
\hline & & & & & 1751.9157 & $-86,189$ & 4 & 13.72 & 99.99 & & & & \\
\hline & & & & & 2026.1360 & $-187,189$ & 4 & 13.16 & 99.99 & 30,2 & 3 & 13.16 & 99.99 \\
\hline
\end{tabular}

Note. Columns are as follows: (1) Quasar name; (2, 3) R.A./decl.; (4) Absorption redshift of LLS; (5) HI column Density; (6) Rest wavelength of transition; (7) Velocity limits $(\mathrm{min} / \mathrm{max})$ for integration relative to $z_{\mathrm{abs}}$; (8) Flag on individual measurement: [0, 1—-standard measurement; 2, 3-lower limit; 4, 5-upper limit]; (9) $\log _{10}$ column density; (10) Standard deviation on $\log _{10} N$. Limits are given a value of 99.99; (11) Ion [atomic number, ionization state]; (12) Flag for the ionic column density [1-standard measurement; 2 -Lower limit; 3 -upper limit]; (13) $\log _{10}$ column density for the ion; (14) Standard deviation on $\log _{10} N_{\text {ion. }}$ Limits are given a value of 99.99 .

(This table is available in its entirety in machine-readable form.)

account for ionization effects when interpreting the results. Figure 13 plots four pairs of ions from the data set, again represented as $\left\{\mathrm{X}^{i} / \mathrm{Y}^{j}\right\}$ with ionization corrections explicitly ignored. The figure also indicates the probable offsets to the ratios if ionization effects were important, as estimated from photoionization calculations (e.g., Prochaska 1999). Similarly, we indicate the likely offsets from differential depletion and the dominant nucleosynthesis channels (Type Ia and Type II enrichment).

The left-hand panels show two measures of $\alpha / \mathrm{Fe}$, a key diagnostic of the relative contributions of Type Ia and Type II SNe nucleosynthesis (Tinsley 1979). Unfortunately, the
$\left\{\mathrm{O}^{0} / \mathrm{Fe}^{+}\right\}$ratios are dominated by lower limits due to the saturation of $\mathrm{O}_{\mathrm{I}} 1302$ and the non-detection of $\mathrm{Fe}$ II transitions. The values are nearly consistent with a solar abundance although there are at least two systems with $\left\{\mathrm{O}^{0} / \mathrm{Fe}^{+}\right\}>+0.3$ dex suggesting an $\alpha$-enhanced gas. Correcting for photoionization effects would only strengthen this conclusion. These two LLSs also exhibit a low metallicity $([\mathrm{O} / \mathrm{H}] \approx-2)$ such that their chemical signature is very similar to that of metal-poor Galactic stars (McWilliam 1997).

Turning to $\left\{\mathrm{Si}^{+} / \mathrm{Fe}^{+}\right\}$, the sample is dominated by measurements exceeding the solar abundance. This includes a non- 

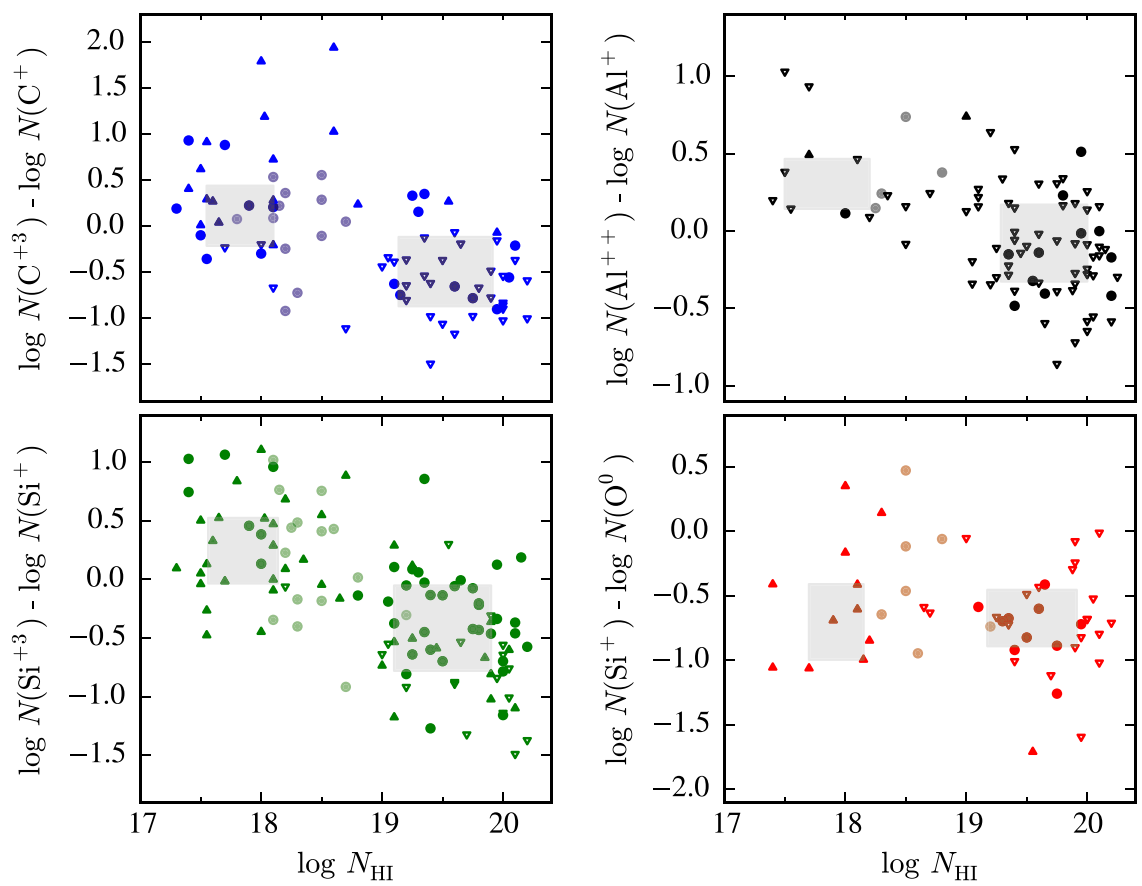

Figure 9. Scatter plots of four ionic ratios that diagnose the ionization state of the LLSs. Gray boxes encompass $50 \%$ of the measurements in two logarithmic $N_{\mathrm{H}}$ intervals: $[17.3,19.0),[19.0,20.3)$. The $\mathrm{C}, \mathrm{Si}$, and $\mathrm{Al}$ ratios show strong evidence that the gas is more highly ionized at low $N_{\mathrm{HI}}$ values. Similarly, the set of $\mathrm{Si}{ }^{+} / \mathrm{O}^{0}$ values exceeding -0.7 dex are indicative of a highly ionized gas. We further emphasize that the $\log \left(\mathrm{Si}^{+} / \mathrm{Si}^{+3}\right) \approx-0.5$ dex values at high $N_{\mathrm{H} \text { I }}$ suggests that this gas is also partially ionized.
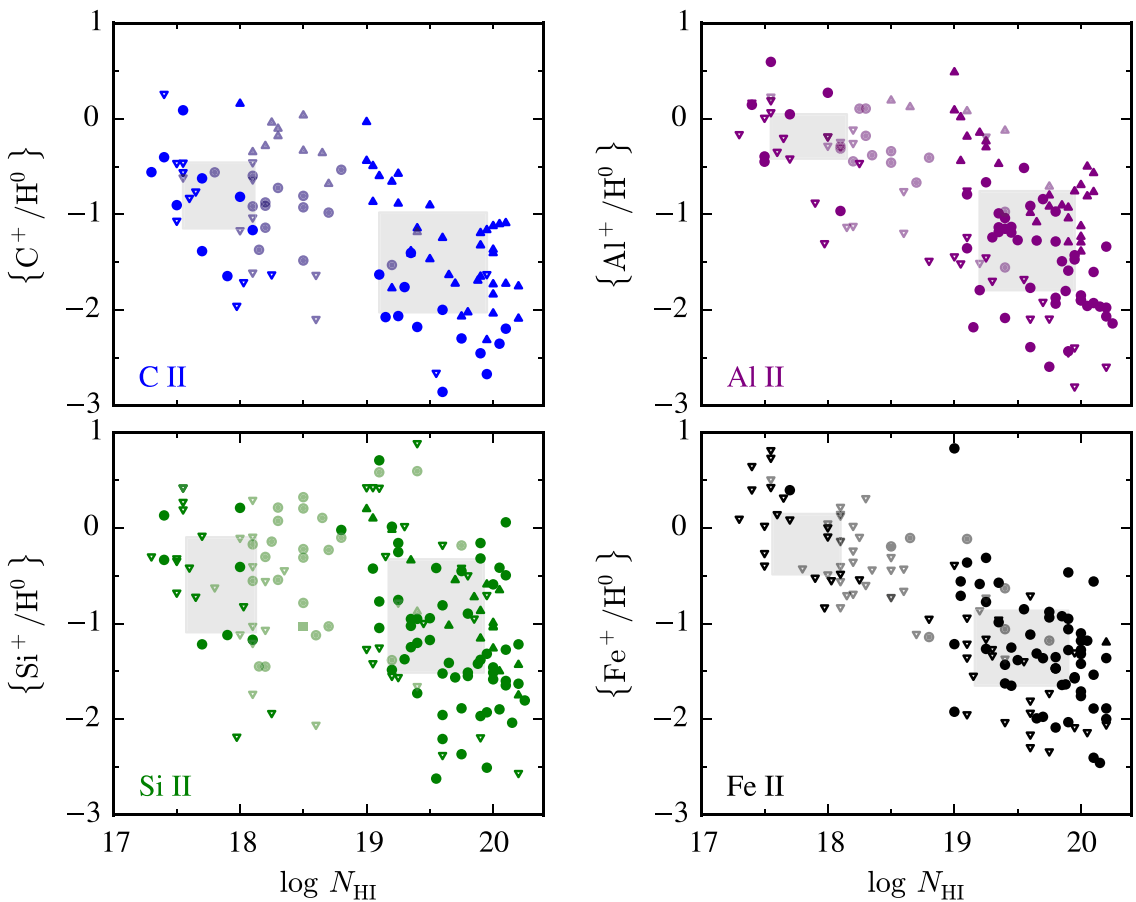

Figure 10. Scatter plots of low-ion column densities relative to $\mathrm{H}$ I, normalized to the solar abundance $\left\{\mathrm{X}^{i} / \mathrm{H}^{0}\right\}$ and plotted against the LLS $N_{\mathrm{H}}$ value. Gray boxes encompass $50 \%$ of the measurements in two logarithmic $N_{\mathrm{H}}$ intervals: $[17.3,19.0),[19.0,20.3)$. If ionization corrections are small, $\left\{\mathrm{X}^{i} / \mathrm{H}^{0}\right\}$ provides an estimate of the logarithmic metal abundance relative to solar. The measurements appear to indicate a declining trend of gas metallicity with increasing $N_{\mathrm{H}}$. We argue, however, that this apparent trend is driven by ionization effects and the set of upper/lower limits at low/high $N_{\mathrm{H} \mathrm{I}}$ values. Furthermore, given the large scatter at all $N_{\mathrm{H} \text { I }}$ values,

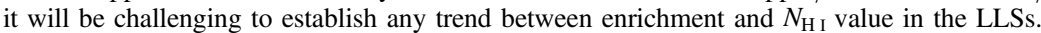

negligible set of measurements with $\left\{\mathrm{Si}^{+} / \mathrm{Fe}^{+}\right\}>+0.5 \mathrm{dex}$, and one may speculate that this represents the metal-enriched ejecta of Type II SNe. The $\left\{\mathrm{Si}^{+} / \mathrm{Fe}^{+}\right\}$ratio, however, is likely to require an ionization correction to accurately estimate $\mathrm{Si} / \mathrm{Fe}$. This could explain, in part, the positive $\left\{\mathrm{Si}^{+} / \mathrm{Fe}^{+}\right\}$values in Figure 13. On the other hand, the highest $\left\{\mathrm{Si}^{+} / \mathrm{Fe}^{+}\right\}$values occur in LLSs with high $N_{\mathrm{H}}$ values where one expects ionization effects to be minimal. ${ }^{12}$ We conclude, therefore, that at least a subset of the LLS population exhibits super-solar

\footnotetext{
${ }^{12}$ Such gas may also experience differential depletion, i.e., elevated $\mathrm{Si} / \mathrm{Fe}$ ratios in the gas phase to the refractory nature of these elements (e.g., Jenkins 2009). If the gas is predominantly ionized, however, the depletion levels may be modest and this effect would be small.
} 


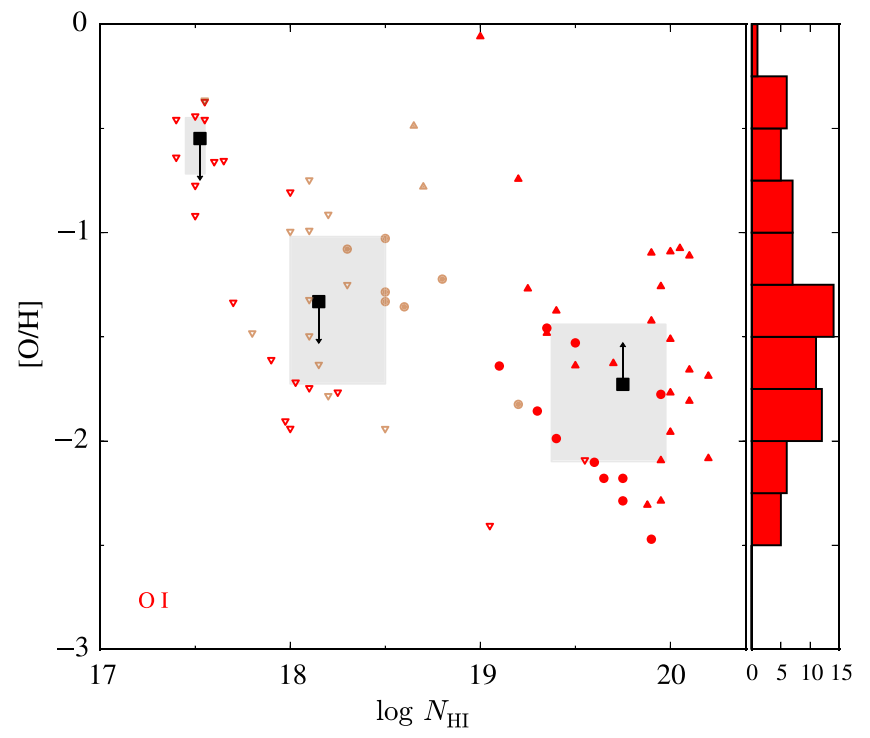

Figure 11. Estimations of the oxygen metallicity in the LLSs where we have assumed that $[\mathrm{O} / \mathrm{H}]=\left\{\mathrm{O}^{0} / \mathrm{H}^{0}\right\}$, i.e., that ionization corrections are small for this ionic ratio (see the text). Gray boxes encompass $50 \%$ of the measurements in three logarithmic $N_{\mathrm{H}}$ intervals: $[17.3,18.0)$, [18.0, 19.0), $[19.0,20.3)$. Similar to the results for other low-ions (Figure 10), there is an apparent decline in $[\mathrm{O} / \mathrm{H}]$ with $N_{\mathrm{HI}}$. This apparent trend, however, is primarily driven by the

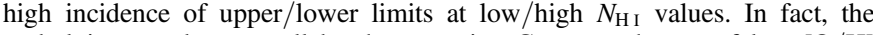
underlying trend may well be the opposite. Compare the set of low $[\mathrm{O} / \mathrm{H}]$ values and upper limits at $N_{\mathrm{HI}} \approx 10^{18} \mathrm{~cm}^{-2}$ with the large set of lower limits at $N_{\mathrm{HI}} \approx 10^{19.7} \mathrm{~cm}^{-2}$.

$\alpha /$ Fe ratios indicative of Type II enrichment, even in higher metallicity gas.
Previous studies of gas in the IGM at $z \sim 2$ have reported an enhanced Si/C abundance (Aguirre et al. 2004). This result was derived statistically from the pixel optical depth method and is sensitive to the assumed model of the EUVB; (see also Simcoe 2011). The results for LLSs offer a mixed picture (Figure 13). There are a handful of positive $\left\{\mathrm{Si}^{+} / \mathrm{C}^{+}\right\}$values up to +0.5 dex, with the highest measurements at low metallicity. On the other hand, the sample is dominated by upper limits (from $\mathrm{C}_{\text {II }} 1334$ saturation) and over half of these have $\left\{\mathrm{Si}^{+} / \mathrm{C}^{+}\right\}<+0.3 \mathrm{dex}$. Once again, photoionization corrections would only strengthen this result. As such, the LLS observations do not appear to exhibit a high enrichment of $\mathrm{Si} / \mathrm{C}$ than that previously inferred for the IGM.

Figure 13 also presents the set of $\left\{\mathrm{Al}^{+} / \mathrm{C}^{+}\right\}$measurements that are not fully compromised by line-saturation. These data are consistent with the lighter element ratios in LLSs having solar relative abundances. The preponderance of upper limits, however, allows that $\mathrm{Al}$ could be under-abundant relative to $\mathrm{C}$.

\subsection{Comparisons}

We have restricted the HD-LLS Sample to systems with $N_{\mathrm{H} \text { I }}<10^{20.3} \mathrm{~cm}^{-2}$ to exclude the DLAs. This was partly motivated by the expectation that the majority of LLSs are predominantly ionized and therefore physically distinct from the neutral gas comprising DLAs. It was also motivated by the desire to examine this optically thick gas separately from the decades of research on the DLAs. Nonetheless, the $N_{\mathrm{H} \text { I }}=10^{20.3} \mathrm{~cm}^{-2}$ criterion is primarily an observationally defined boundary and one may gain insight into the nature of the LLSs through a combined comparison. Such analysis has been performed previously by Peroux et al. (2005) and Som et al. (2013).
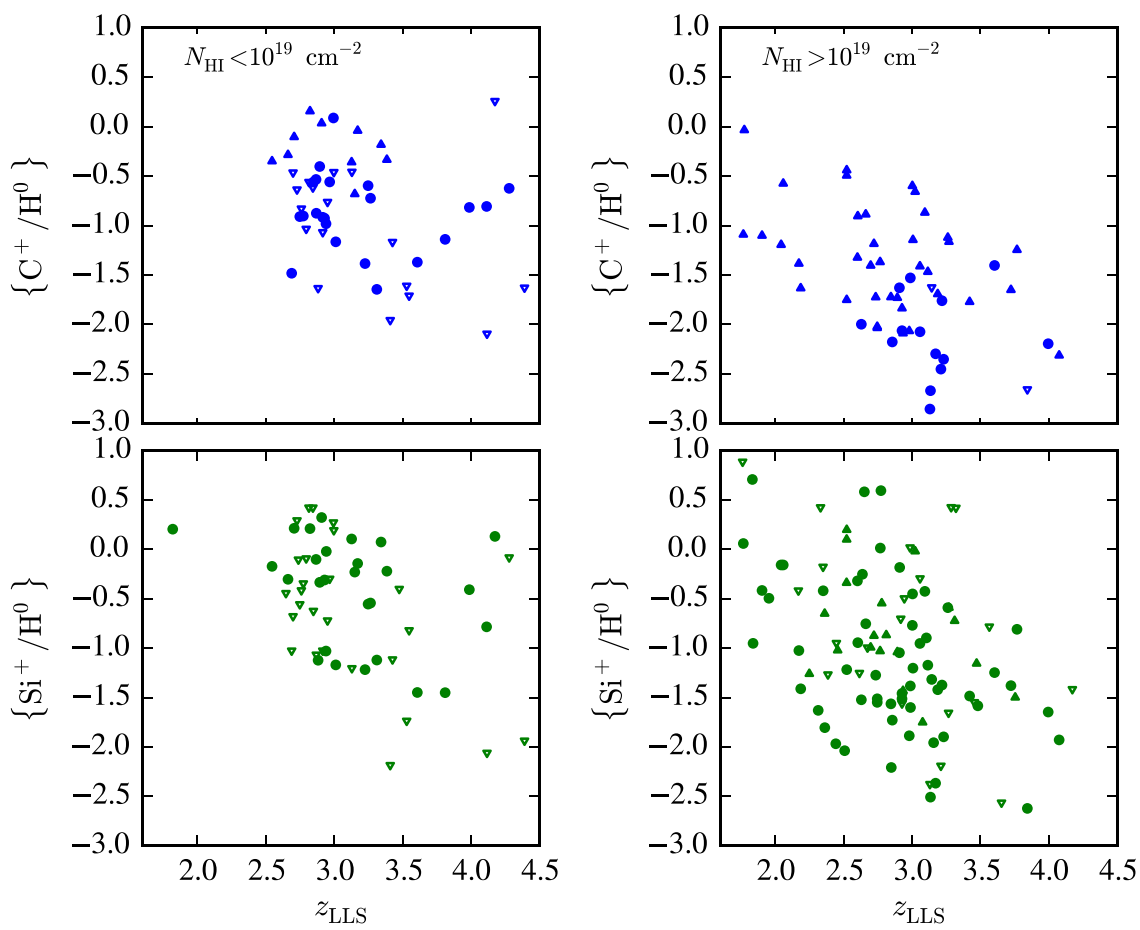

Figure 12. Comparison of $\left\{\mathrm{X}^{i} / \mathrm{H}^{0}\right\}$ measurements for $\mathrm{C}^{+}$and $\mathrm{Si}^{+}$against absorption redshift. Left-hand panels are for the LLSs with $N_{\mathrm{H}}<10^{19} \mathrm{~cm}{ }^{-2}$ and the righthand panels are for the SLLS population. All of the data show evidence for a declining enrichment with increasing redshift, although this assertion is statistically significant (>99\% c.l.) only for the SLLS sample. The absence of low values at $z \approx 2$ implies a reduced incidence of near-pristine gas with high $\mathrm{H}$ i columns at that epoch. 

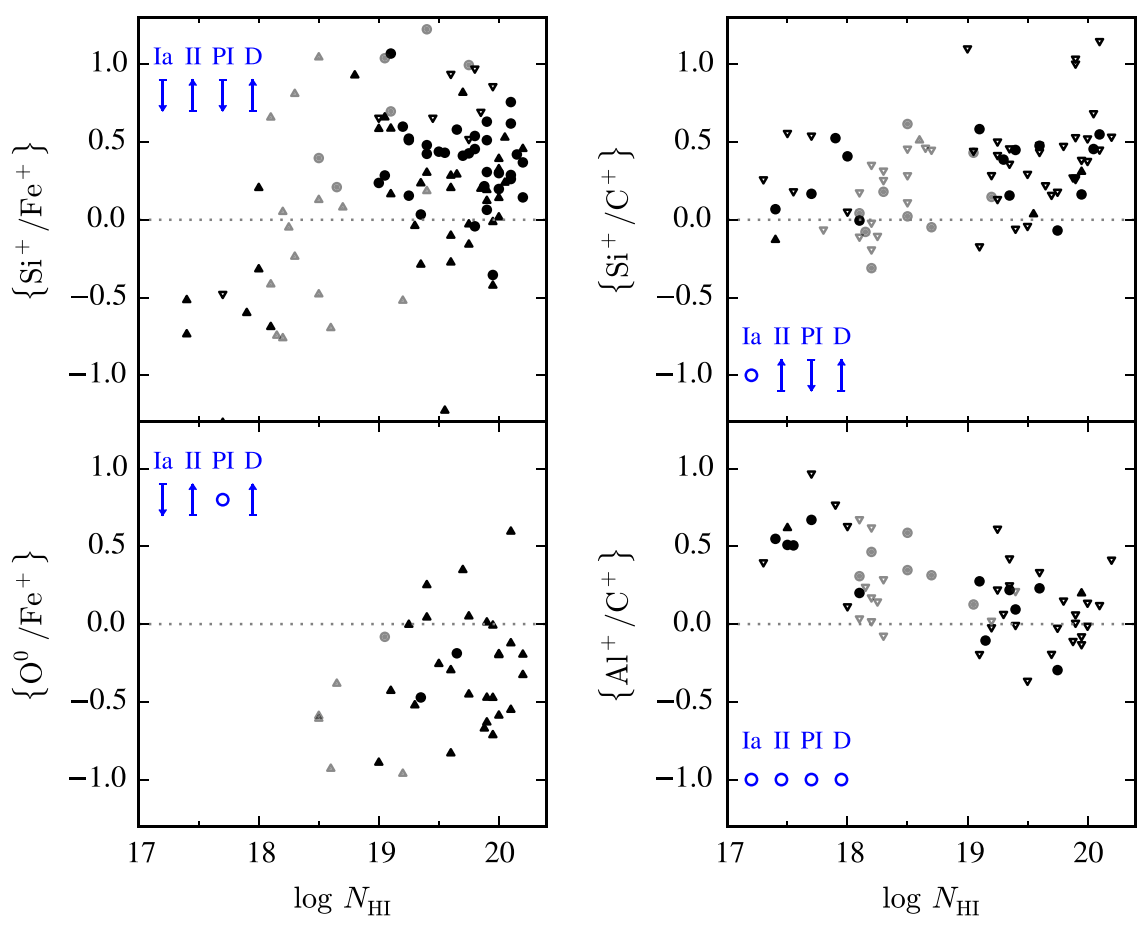

Figure 13. Scatter plots of a series of ionic ratios, normalized to the solar relative abundances against the $N_{\mathrm{H}}$ values of the LLSs. The left two panels show ratios related to the $\alpha / \mathrm{Fe}$ abundance. Despite the preponderance of lower limits (especially for $\left\{\mathrm{O}^{0} / \mathrm{Fe}^{+}\right\}$), and concerns on the ionization corrections, we tentatively conclude that the LLSs exhibit super-solar $\alpha / \mathrm{Fe}$ ratios, especially at large $N_{\mathrm{HI}}$ values. The measurements in the upper-right panel indicate that $\mathrm{Si} / \mathrm{C}$ is possibly enhanced by a few 0.1 dex relative to solar although the majority of the sample is consistent with $[\mathrm{Si} / \mathrm{C}]=0$. Similarly, the $\left\{\mathrm{Al}^{+} / \mathrm{C}^{+}\right\}$measurements are roughly consistent with the solar relative abundance or possibly sub-solar. In each panel, we indicate the expected offsets to the measurements that would be due to Type Ia (Ia) nucleosynthesis, Type II (II) nucleosynthesis, photoionization (PI), and differential depletion (D). Circles indicate a small or unknown impact.

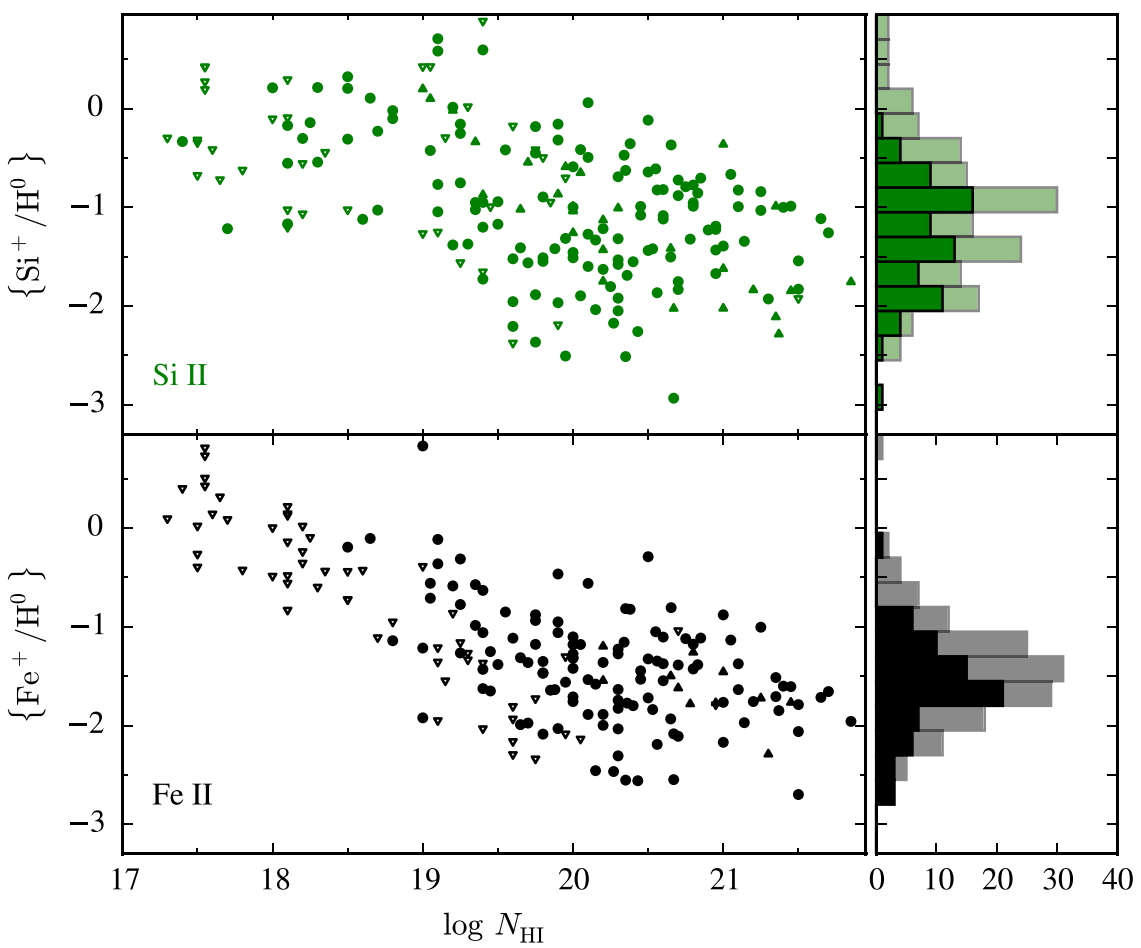

Figure 14. Comparison of the $\left\{\mathrm{Si}^{+} / \mathrm{H}^{0}\right\}$ and $\left\{\mathrm{Fe}^{+} / \mathrm{H}^{0}\right\}$ measurements for the LLSs and DLAs. The latter are drawn from the abundance compilation of Rafelski et al. (2012). For both ions, the DLAs show a continuous extension of the measurements observed in the LLSs. The right-hand panels compare the distributions of the DLAs (darker) against those for the SLLSs (lighter), and emphasize the commonality between the two data sets. In each case, we have treated upper and lower limit estimates as values. 


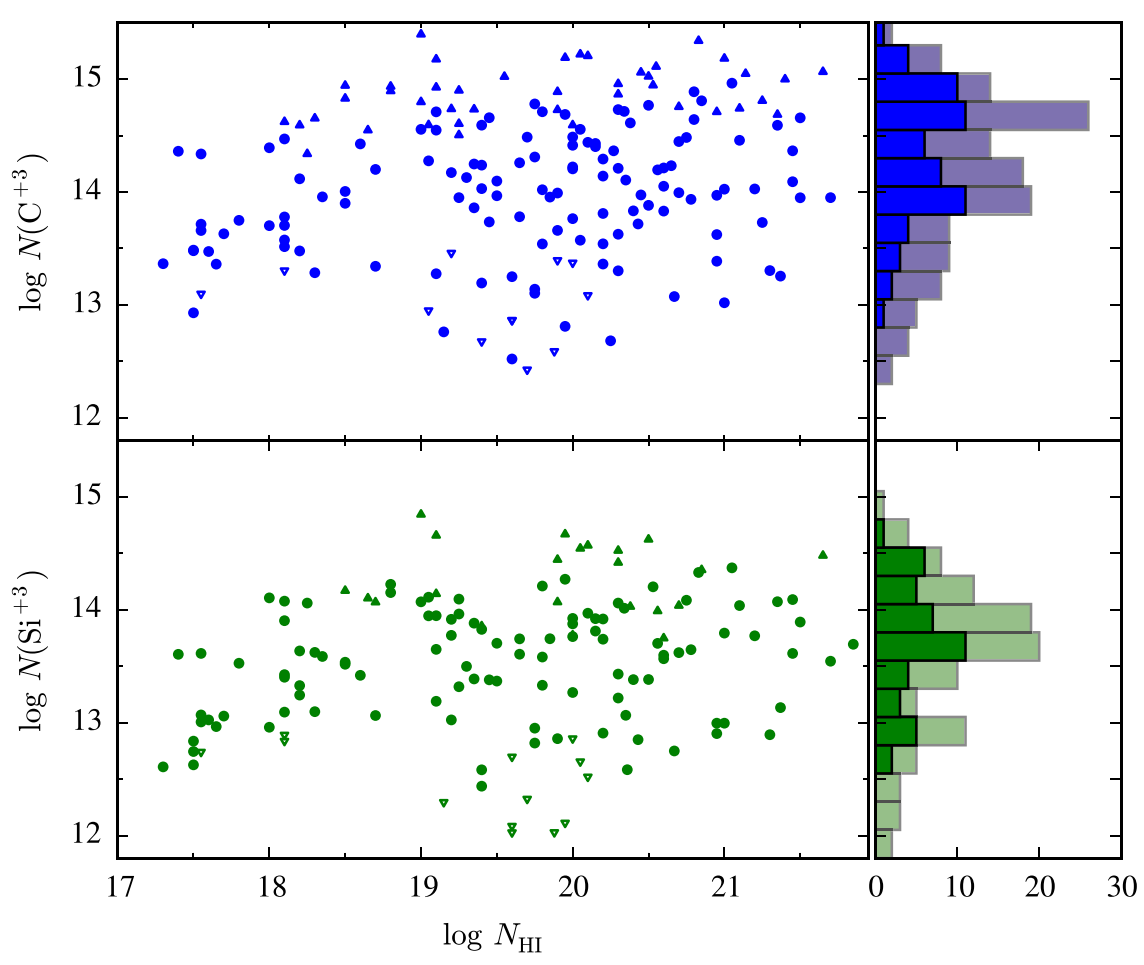

Figure 15. Comparison of the high-ion column densities measured for the LLS and a representative set of DLAs (drawn from Rafelski et al. 2012; Neeleman et al. 2013). Similar to the low-ion abundances, the measurements show a continuous transition from the LLS regime to higher $N_{\mathrm{HI}}$ values. As such, there is substantial overlap in the distribution of measurements and limits (right-hand panels compare the values for DLAs [darker] and SLLSs [lighter]). One notable and surprising difference is that the SLLSs show a higher incidence of low columns of $\mathrm{C}^{+3}$ and $\mathrm{Si}^{+3}$. Despite tracing predominantly neutral gas, the DLAs also mark a reservoir of highly ionized gas that frequently exceeds the medium encompassing the LLSs.

We consider two such comparisons here. Figure 14 presents the $\left\{\mathrm{Si}^{+} / \mathrm{H}^{0}\right\}$ and $\left\{\mathrm{Fe}^{+} / \mathrm{H}^{0}\right\}$ measurements for the HD-LLS Sample together with measurements from the sample of DLAs of Rafelski et al. (2012). For both data sets, we have restricted to $z_{\mathrm{abs}}=[1.6,3.3]$ to minimize trends related to redshift evolution. To zeroth order, the DLA measurements extend in a roughly continuous manner from the measurements of the LLSs. Indeed, comparing the samples of DLA measurements with the SLLSs (taking limits at their values), one observes overlapping distributions with similar median values. The only notable distinction, perhaps, is the small set of LLSs with $N_{\mathrm{H} \text { I }} \approx 10^{19} \mathrm{~cm}^{-2}$ and high $\left\{\mathrm{X}^{i} / \mathrm{H}^{0}\right\}$ values (exceeding $0 \mathrm{dex}$ for $\mathrm{Si}^{+}$). This suggests a higher incidence of highly enriched gas in the LLS, although we caution it could be partly an effect of ionization. The dispersion in the measurements is also larger for the LLSs, and is likely higher than suggested by the Figure given the preponderance of upper/lower limits for the LLS/DLA.

Turning to the higher ionization states, Figure 15 presents the $\mathrm{C}^{+3}$ and $\mathrm{Si}^{+3}$ column densities from the LLSs and DLAs. Once again, the DLA distribution extends in a nearly continuous manner from the upper end of the LLS data and the column density distributions for the SLLSs and DLAs are similar. Together, Figures 14 and 15 lend support to scenarios that envision LLSs as the outer layers of gas surrounding DLAs, i.e., these systems frequently sample the same structures. Such physical associations may be examined by studying DLAs and LLSs along pairs of quasar sightlines, an active area of research (Ellison et al. 2007; Fumagalli et al. 2014; Rubin et al. 2014).
Examining the high-ion comparison further, there is at least one important distinction: the LLSs and especially the SLLSs show a much higher incidence of low $N\left(\mathrm{C}^{+3}\right)$ and $N\left(\mathrm{Si}^{+3}\right)$ values. This is unexpected given that (i) the LLSs trace highly ionized gas; (ii) the DLAs trace predominantly neutral gas that is physically distinct from the high-ions (Wolfe \& Prochaska 2000; Prochaska et al. 2008a). The results presented here indicate that the gas layers giving rise to DLAs are embedded in a reservoir of highly ionized gas that frequently exceeds the typical surface density in LLSs. This follows previous work which has inferred high quantities of both neutral and ionized gas for the DLAs (Fox et al. 2007; Lehner et al. 2014). It further suggests that high-ions more closely trace higher density regions in the universe and/or may reflect a difference in the masses of the dark matter halos hosting LLSs and DLAs.

Lastly, we have compared our measurements against the small set of literature values for SLLSs at $z>2$ (DessaugesZavadsky et al. 2003; Som et al. 2013). The low-ion column densities are typically larger in those publications, consistent with the higher $N_{\mathrm{H} \text { I }}$ values of the SLLSs that were sampled.

\section{SUMMARY}

We have constructed a sample of 157 LLSs at $z \sim 2-4$ observed at high-dispersion with spectrometers on the Keck and Magellan telescopes which constitute the HD-LLS Sample. In this manuscript, we present the complete sample and present column density measurements of $\mathrm{HI}_{\mathrm{I}}$ and associated metal absorption. For the latter, analysis was restricted to transitions redward of the $\operatorname{Ly} \alpha$ forest and has focused on commonly 


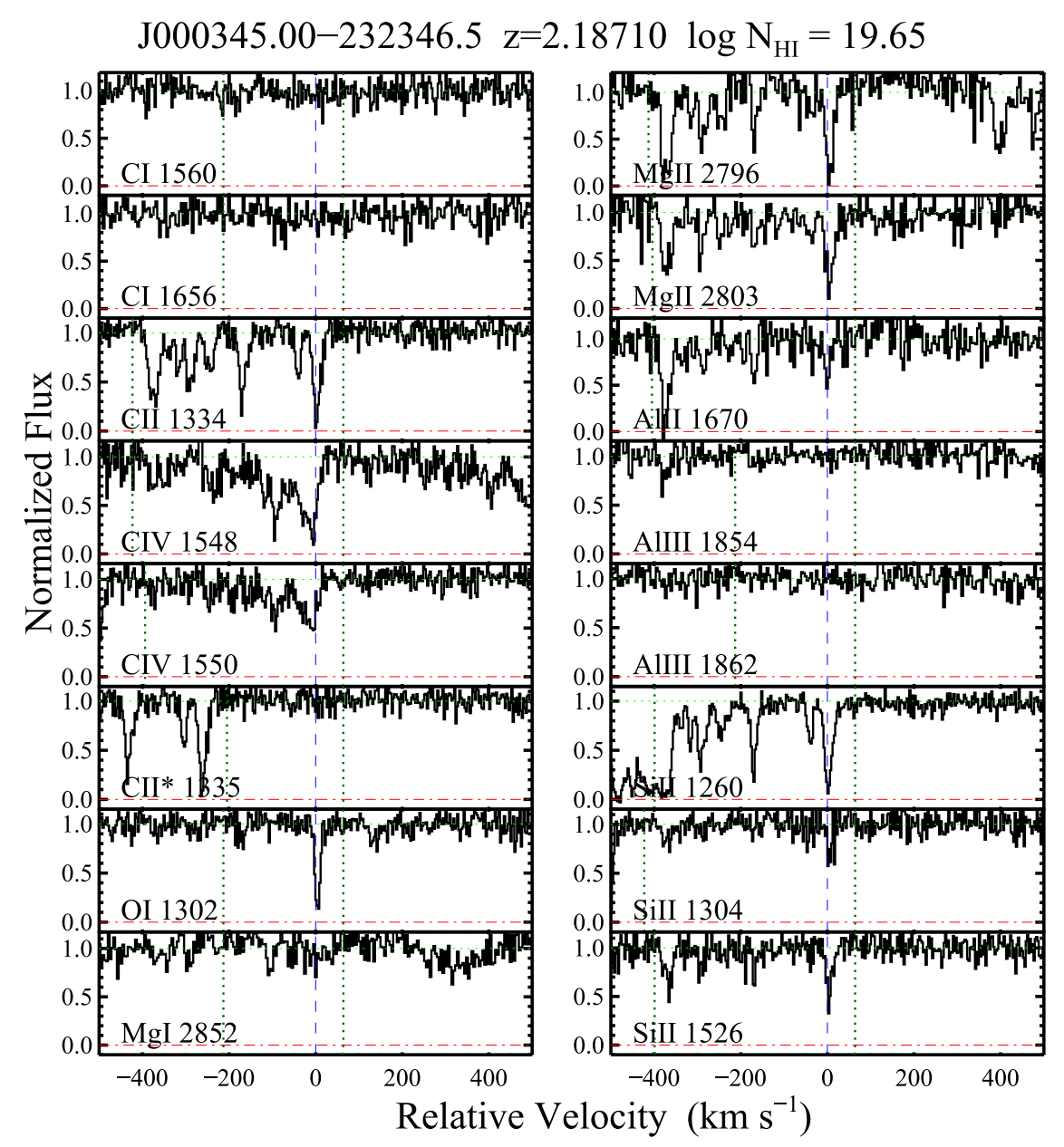

Figure 16. Velocity plots for the HD-LLS Sample.

(The complete figure set (260 images) is available.)

detected species. These measurements and the associated spectra are made available online with this publication. ${ }^{13}$ This constitutes, by roughly an order of magnitude, the largest highredshift sample of LLS analyzed in this manner.

We have explored empirical trends in the column density measurements and report statistically significant (>99.99\%) correlations between the low-ion (e.g., $\mathrm{Si}^{+}, \mathrm{C}^{+}$) columns and $N_{\mathrm{H} \text { I }}$. High-ion species $\left(\mathrm{Si}^{+3}, \mathrm{C}^{+3}\right)$ are detected in nearly all LLSs and their column densities also correlated with $N_{\mathrm{HI}}$. Examining ionic ratios sensitive to the ionization state (e.g., $\mathrm{C}^{+3} / \mathrm{C}^{+}, \mathrm{Si}^{+3} / \mathrm{Si}^{+}$), we conclude that the LLSs are predominantly ionized with more highly ionized gas in lower $N_{\mathrm{H} \text { I }}$ systems.

Ratios of low-ion column densities to $N_{\mathrm{H}}$ indicate a wide spread in metal-enrichment within the LLSs, likely spanning four orders of magnitude. Only a small subset $(\lesssim 5 \%)$ of the HD-LLS Sample have no positive detections of associated metals, consistent with primordial abundances. None of the LLSs with $N_{\mathrm{H}} \geqslant 10^{19.2} \mathrm{~cm}^{-2}$ are "metal-free." We conclude that a very high percentage of high-density gas at $z \sim 3$ was previously enriched to $\gtrsim 1 / 1000$ solar abundance. The HDLLS Sample also exhibits a small subset $(\sim 10 \%)$ of LLSs that have solar or super-solar enrichment. These likely represent the most enriched gas reservoirs in the high redshift universe.

\footnotetext{
${ }^{13}$ http://www.ucolick.org/ xavier/LLS
}

Lastly, we have examined several ionic ratios that are sensitive to the nucleosynthetic enrichment history of the gas. The preponderance of elevated $\mathrm{Si}^{+} / \mathrm{Fe}^{+}$and $\mathrm{O}^{0} / \mathrm{Fe}^{+}$measurements suggest the LLSs have an $\alpha$-enhancement characteristic of Type II nucleosynthesis. In contrast, the $\mathrm{Si}^{+} / \mathrm{C}^{+}$and $\mathrm{Al}^{+} / \mathrm{C}^{+}$ratios are consistent with solar relative abundances.

Future manuscripts on the HD-LLS Sample will: (i) study the metallicity distribution of the LLSs accounting for ionization effects and will estimate the contribution of optically thick gas to the cosmic metal budget; (ii) examine the kinematic characteristics to constrain the physical origin of the gas; (iii) offer constraints on the $N_{\mathrm{H}}$ frequency distribution for optically thick gas.

J.X.P. was supported by NSF grants AST-1010004 and AST-1412981. M.F. acknowledges support by the Science and Technology Facilities Council, grant number ST/L00075X/1. We thank Claude-André Faucher-Giguerre for kindly providing his continuum fits to MIKE spectra. We acknowledge the contributions of Wal Sargent and Brian Penprase in collecting a portion of the ESI data and Arthur M. Wolfe, Marcel Neeleman, and Marc Rafelski for their contributions to the Keck observations.

Much of the data presented herein were obtained at the W. M. Keck Observatory, which is operated as a scientific partnership among the California Institute of Technology, the 
University of California, and the National Aeronautics and Space Administration. The Observatory was made possible by the generous financial support of the W. M. Keck Foundation. Some of the Keck data were obtained through the NSF Telescope System Instrumentation Program (TSIP), supported by AURA through the NSF under AURA Cooperative Agreement AST 01-32798 as amended.

\section{APPENDIX MEASUREMENTS FOR INDIVIDUAL LLS}

Table 6 lists measurements for all of the metal-line transitions analyzed in this manuscript and figures showing velocity plots are provided in the online material (Figure 16 shows one example). The analysis was restricted to lines outside the $\operatorname{Ly} \alpha$ forest and those lines that are not severely blended with another feature or compromised by sky-subtraction residuals.

\section{REFERENCES}

Aguirre, A., Hernquist, L., Schaye, J., et al. 2001, ApJ, 560, 599

Aguirre, A., Schaye, J., Kim, T.-S., et al. 2004, ApJ, 602, 38

Asplund, M., Grevesse, N., Sauval, A. J., \& Scott, P. 2009, ARA\&A, 47, 481

Berg, T. A. M., Neeleman, M., Prochaska, J. X., Ellison, S. L., \& Wolfe, A. M. 2014, PASP, 127, 167

Bernstein, R., Shectman, S. A., Gunnels, S. M., Mochnacki, S., \& Athey, A. E. 2003, Proc. SPIE, 4841, 1694

Bochanski, J. J., Hennawi, J. F., Simcoe, R. A., et al. 2009, PASP, 121, 1409 Bouché, N., Lehnert, M. D., \& Péroux, C. 2006, MNRAS, 367, L16

Burles, S., \& Tytler, D. 1998, ApJ, 499, 699

Chen, H., Helsby, J. E., Gauthier, J., et al. 2010, ApJ, 714, 1521

Dekel, A., Birnboim, Y., Engel, G., et al. 2009, Natur, 457, 451

Dessauges-Zavadsky, M., Péroux, C., Kim, T.-S., D’Odorico, S., \& McMahon, R. G. 2003, MNRAS, 345, 447

Ellison, S. L., Hennawi, J. F., Martin, C. L., \& Sommer-Larsen, J. 2007, MNRAS, 378, 801

Faucher-Giguere, C.-A., Hopkins, P. F., Keres, D., et al. 2014, MNRAS, 449, 987

Faucher-Giguère, C.-A., \& Kereš, D. 2011, MNRAS, 412, L118

Faucher-Giguère, C.-A., Prochaska, J. X., Lidz, A., Hernquist, L., \& Zaldarriaga, M. 2008, ApJ, 681, 831

Fox, A. J., Petitjean, P., Ledoux, C., \& Srianand, R. 2007, A\&A, 465, 171

Fumagalli, M., Hennawi, J. F., Prochaska, J. X., et al. 2014, ApJ, 780, 74

Fumagalli, M., O’Meara, J. M., \& Prochaska, J. X. 2011a, Sci, 334, 1245

Fumagalli, M., O’Meara, J. M., Prochaska, J. X., \& Worseck, G. 2013, ApJ, 775,78

Fumagalli, M., Prochaska, J. X., Kasen, D., et al. 2011b, MNRAS, 418, 1796

Hennawi, J. F., \& Prochaska, J. X. 2007, ApJ, 655, 735

Jenkins, E. B. 2009, ApJ, 700, 1299

Kereš, D., Katz, N., Weinberg, D. H., \& Davé, R. 2005, MNRAS, 363, 2

Lehner, N., Howk, J. C., Tripp, T. M., et al. 2013, ApJ, 770, 138

Lehner, N., O’Meara, J. M., Fox, A. J., et al. 2014, ApJ, 788, 119

Marshall, J. L., Burles, S., Thompson, I. B., et al. 2008, Proc. SPIE, 7014, 54

McWilliam, A. 1997, ARA\&A, 35, 503
Neeleman, M., Wolfe, A. M., Prochaska, J. X., \& Rafelski, M. 2013, ApJ, 769,54

O’Meara, J. M., Burles, S., Prochaska, J. X., et al. 2006, ApJL, 649, L61

O’Meara, J. M., Lehner, N., Howk, J. C., et al. 2015, AJ, in press (astro-ph/ 1505.03529)

O’Meara, J. M., Prochaska, J. X., Burles, S., et al. 2007, ApJ, 656, 666

O’Meara, J. M., Prochaska, J. X., Worseck, G., Chen, H.-W., \& Madau, P. 2013, ApJ, 765, 137

Peeples, M. S., Werk, J. K., Tumlinson, J., et al. 2014, ApJ, 786, 54

Penprase, B. E., Prochaska, J. X., Sargent, W. L. W., Toro-Martinez, I., \& Beeler, D. J. 2010, ApJ, 721, 1

Péroux, C., Dessauges-Zavadsky, M., D’Odorico, S., Sun Kim, T., \& McMahon, R. G. 2005, MNRAS, 363, 479

Prochaska, J. X. 1999, ApJL, 511, L71

Prochaska, J. X., Chen, H.-W., Wolfe, A. M., Dessauges-Zavadsky, M., \& Bloom, J. S. 2008a, ApJ, 672, 59

Prochaska, J. X., Gawiser, E., Wolfe, A. M., Castro, S., \& Djorgovski, S. G. 2003a, ApJL, 595, L9

Prochaska, J. X., Gawiser, E., Wolfe, A. M., Cooke, J., \& Gelino, D. 2003b, ApJS, 147, 227

Prochaska, J. X., \& Hennawi, J. F. 2009, ApJ, 690, 1558

Prochaska, J. X., Hennawi, J. F., \& Herbert-Fort, S. 2008b, ApJ, 675, 1002

Prochaska, J. X., Hennawi, J. F., Lee, K.-G., et al. 2013, ApJ, 776, 136

Prochaska, J. X., Lau, M. W., \& Hennawi, J. F. 2014a, ApJ, 796, 140

Prochaska, J. X., Madau, P., O’Meara, J. M., \& Fumagalli, M. 2014b, MNRAS, 438, 476

Prochaska, J. X., O’Meara, J. M., Herbert-Fort, S., et al. 2006, ApJL, 648, L97

Prochaska, J. X., O’Meara, J. M., \& Worseck, G. 2010, ApJ, 718, 392

Prochaska, J. X., \& Wolfe, A. M. 1997, ApJ, 487, 73

Prochaska, J. X., Wolfe, A. M., Howk, J. C., et al. 2007, ApJS, 171, 29

Prochaska, J. X., Worseck, G., \& O’Meara, J. M. 2009, ApJL, 705, L113

Prochaska, J. X., Wolfe, A. M., Tytler, D., et al. 2001, ApJS, 137, 21

Prochter, G. E., Prochaska, J. X., O'Meara, J. M., Burles, S., \& Bernstein, R. A. 2010, ApJ, 708, 1221

Rafelski, M., Wolfe, A. M., Prochaska, J. X., Neeleman, M., \& Mendez, A. J. 2012, ApJ, 755, 89

Ribaudo, J., Lehner, N., \& Howk, J. C. 2011, ApJ, 736, 42

Rubin, K. H. R., Hennawi, J. F., Prochaska, J. X., et al. 2014, ApJ, 808, 38

Rudie, G. C., Steidel, C. C., Trainor, R. F., et al. 2012, ApJ, 750, 67

Sargent, W. L. W., Steidel, C. C., \& Boksenberg, A. 1989, ApJS, 69, 703

Savage, B. D., \& Sembach, K. R. 1991, ApJ, 379, 245

Schaye, J., Aguirre, A., Kim, T.-S., et al. 2003, ApJ, 596, 768

Sheinis, A. I., Bolte, M., Epps, H. W., et al. 2002, PASP, 114, 851

Simcoe, R. A. 2011, ApJ, 738, 159

Sofia, U. J., \& Jenkins, E. B. 1998, ApJ, 499, 951

Som, D., Kulkarni, V. P., Meiring, J., et al. 2013, MNRAS, 435, 1469

Songaila, A., \& Cowie, L. L. 2010, ApJ, 721, 1448

Steidel, C. C. 1990, ApJS, 74, 37

Steidel, C. C., Erb, D. K., Shapley, A. E., et al. 2010, ApJ, 717, 289

Storrie-Lombardi, L. J., McMahon, R. G., Irwin, M. J., \& Hazard, C. 1994, ApJL, 427, L13

Tinsley, B. M. 1979, ApJ, 229, 1046

Tytler, D. 1982, Natur, 298, 427

Vogt, S. S., Allen, S. L., Bigelow, B. C., et al. 1994, Proc. SPIE, 2198, 362

Werk, J. K., Prochaska, J. X., Thom, C., et al. 2013, ApJS, 204, 17

Wolfe, A. M., \& Prochaska, J. X. 2000, ApJ, 545, 591

Worseck, G., Prochaska, J. X., O’Meara, J. M., et al. 2014, MNRAS, 445, 1745

Zafar, T., Popping, A., \& Péroux, C. 2013, A\&A, 556, A140 